\title{
Learning Scheduling Algorithms for Data Processing Clusters
}

\author{
Hongzi Mao, Malte Schwarzkopf, Shaileshh Bojja Venkatakrishnan, Zili Meng ${ }^{\star}$, Mohammad Alizadeh \\ MIT Computer Science and Artificial Intelligence Laboratory ${ }^{\star}$ Tsinghua University \\ \{hongzi,malte,bjjvnkt,alizadeh\}@csail.mit.edu,mengzl15@mails.tsinghua.edu.cn
}

\begin{abstract}
Efficiently scheduling data processing jobs on distributed compute clusters requires complex algorithms. Current systems use simple, generalized heuristics and ignore workload characteristics, since developing and tuning a scheduling policy for each workload is infeasible. In this paper, we show that modern machine learning techniques can generate highly-efficient policies automatically.

Decima uses reinforcement learning (RL) and neural networks to learn workload-specific scheduling algorithms without any human instruction beyond a high-level objective, such as minimizing average job completion time. However, off-the-shelf RL techniques cannot handle the complexity and scale of the scheduling problem. To build Decima, we had to develop new representations for jobs' dependency graphs, design scalable RL models, and invent RL training methods for dealing with continuous stochastic job arrivals.

Our prototype integration with Spark on a 25-node cluster shows that Decima improves average job completion time by at least $21 \%$ over hand-tuned scheduling heuristics, achieving up to $2 \times$ improvement during periods of high cluster load.
\end{abstract}

CCS Concepts: Software and its engineering $\rightarrow$ Scheduling; Networks $\rightarrow$ Network resources allocation; Computing methodologies $\rightarrow$ Reinforcement learning

Keywords: resource management, job scheduling, reinforcement learning

\section{ACM Reference Format:}

Hongzi Mao, Malte Schwarzkopf, Shaileshh Bojja Venkatakrishnan, Zili Meng and Mohammad Alizadeh. 2019. Learning Scheduling Algorithms for Data Processing Clusters. In SIGCOMM '19, August 19-23, 2019, Beijing, China. ACM, Beijing, China, 19 pages. https://doi.org/10.1145/3341302.3342080

\section{Introduction}

Efficient utilization of expensive compute clusters matters for enterprises: even small improvements in utilization can save millions of dollars at scale [11, §1.2]. Cluster schedulers are key to realizing these savings. A good scheduling policy packs work tightly to reduce fragmentation [34, 36, 76], prioritizes jobs according to high-level metrics such as user-perceived latency [77], and avoids inefficient configurations [28]. Current cluster schedulers rely on heuristics that prioritize generality, ease of understanding, and straightforward implementation over achieving the ideal performance on a specific workload. By using general heuristics like fair scheduling [8,31], shortest-job-first,

Permission to make digital or hard copies of all or part of this work for personal or classroom use is granted without fee provided that copies are not made or distributed for profit or commercial advantage and that copies bear this notice and the full citation on the first page. Copyrights for components of this work owned by others than ACM must be honored. Abstracting with credit is permitted. To copy otherwise, or republish, to post on servers or to redistribute to lists, requires prior specific permission and/or a fee. Request permissions from permissions@ acm.org.

SIGCOMM '19, August 19-23, 2019, Beijing, China

(C) 2019 Association for Computing Machinery.

ACM ISBN 978-1-4503-5956-6/19/08 _\$15.00

https://doi.org/10.1145/3341302.3342080 and simple packing strategies [34], current systems forego potential performance optimizations. For example, widely-used schedulers ignore readily available information about job structure (i.e., internal dependencies) and efficient parallelism for jobs' input sizes. Unfortunately, workload-specific scheduling policies that use this information require expert knowledge and significant effort to devise, implement, and validate. For many organizations, these skills are either unavailable, or uneconomic as the labor cost exceeds potential savings.

In this paper, we show that modern machine-learning techniques can help side-step this trade-off by automatically learning highly efficient, workload-specific scheduling policies. We present Decima ${ }^{1}$, a general-purpose scheduling service for data processing jobs with dependent stages. Many systems encode job stages and their dependencies as directed acyclic graphs (DAGs) [10, 19, 42, 80]. Efficiently scheduling DAGs leads to hard algorithmic problems whose optimal solutions are intractable [36]. Given only a high-level goal (e.g., minimize average job completion time), Decima uses existing monitoring information and past workload logs to automatically learn sophisticated scheduling policies. For example, instead of a rigid fair sharing policy, Decima learns to give jobs different shares of resources to optimize overall performance, and it learns job-specific parallelism levels that avoid wasting resources on diminishing returns for jobs with little inherent parallelism. The right algorithms and thresholds for these policies are workload-dependent, and achieving them today requires painstaking manual scheduler customization.

Decima learns scheduling policies through experience using modern reinforcement learning (RL) techniques. RL is well-suited to learning scheduling policies because it allows learning from actual workload and operating conditions without relying on inaccurate assumptions. Decima encodes its scheduling policy in a neural network trained via a large number of simulated experiments, during which it schedules a workload, observes the outcome, and gradually improves its policy. However, Decima's contribution goes beyond merely applying off-the-shelf RL algorithms to scheduling: to successfully learn high-quality scheduling policies, we had to develop novel data and scheduling action representations, and new RL training techniques.

First, cluster schedulers must scale to hundreds of jobs and thousands of machines, and must decide among potentially hundreds of configurations per job (e.g., different levels of parallelism). This leads to much larger problem sizes compared to conventional RL applications (e.g., game-playing [61, 70], robotics control [51, 67]), both in the amount of information available to the scheduler (the state space), and the number of possible choices it must consider (the $a c$ tion space) ${ }^{2}$ We designed a scalable neural network architecture that combines a graph neural network $[12,23,24,46]$ to process job and cluster information without manual feature engineering, and a policy

\footnotetext{
${ }^{1}$ In Roman mythology, Decima measures threads of life and decides their destinies. ${ }^{2}$ For example, the state of the game of Go [71] can be represented by $19 \times 19=361$ numbers, which also bound the number of legal moves per turn.
} 
network that makes scheduling decisions. Our neural networks reuse a small set of building block operations to process job DAGs, irrespective of their sizes and shapes, and to make scheduling decisions, irrespective of the number of jobs or machines. These operations are parameterized functions learned during training, and designed for the scheduling domain - e.g., ensuring that the graph neural network can express properties such as a DAG's critical path. Our neural network design substantially reduces model complexity compared to naive encodings of the scheduling problem, which is key to efficient learning, fast training, and low-latency scheduling decisions.

Second, conventional RL algorithms cannot train models with continuous streaming job arrivals. The randomness of job arrivals can make it impossible for RL algorithms to tell whether the observed outcome of two decisions differs due to disparate job arrival patterns, or due to the quality the policy's decisions. Further, RL policies necessarily make poor decisions in early stages of training. Hence, with an unbounded stream of incoming jobs, the policy inevitably accumulates a backlog of jobs from which it can never recover. Spending significant training time exploring actions in such situations fails to improve the policy. To deal with the latter problem, we terminate training "episodes" early in the beginning, and gradually grow their length. This allows the policy to learn to handle simple, short job sequences first, and to then graduate to more challenging arrival sequences. To cope with the randomness of job arrivals, we condition training feedback on the actual sequence of job arrivals experienced, using a recent technique for RL in environments with stochastic inputs [55]. This isolates the contribution of the scheduling policy in the feedback and makes it feasible to learn policies that handle stochastic job arrivals.

We integrated Decima with Spark and evaluated it in both an experimental testbed and on a workload trace from Alibaba's production clusters $[6,52] .^{3}$ Our evaluation shows that Decima outperforms existing heuristics on a 25-node Spark cluster, reducing average job completion time of TPC-H query mixes by at least $21 \%$. Decima's policies are particularly effective during periods of high cluster load, where it improves the job completion time by up to $2 \times$ over existing heuristics. Decima also extends to multi-resource scheduling of CPU and memory, where it improves average job completion time by $32-43 \%$ over prior schemes such as Graphene [36].

In summary, we make the following key contributions:

(1) A scalable neural network design that can process DAGs of arbitrary shapes and sizes, schedule DAG stages, and set efficient parallelism levels for each job (\$5.1-\$5.2).

(2) A set of RL training techniques that for the first time enable training a scheduler to handle unbounded stochastic job arrival sequences $(\$ 5.3)$.

(3) Decima, the first RL-based scheduler that schedules complex data processing jobs and learns workload-specific scheduling policies without human input, and a prototype implementation of it (\$6).

(4) An evaluation of Decima in simulation and in a real Spark cluster, and a comparison with state-of-the-art scheduling heuristics (§7).

\section{Motivation}

Data processing systems and query compilers such as Hive, Pig, SparkSQL, and DryadLINQ create DAG-structured jobs, which consist of processing stages connected by input/output dependencies (Figure 1).

\footnotetext{
${ }^{3}$ We used an earlier version of Alibaba's public cluster-trace-v2018 trace.
}

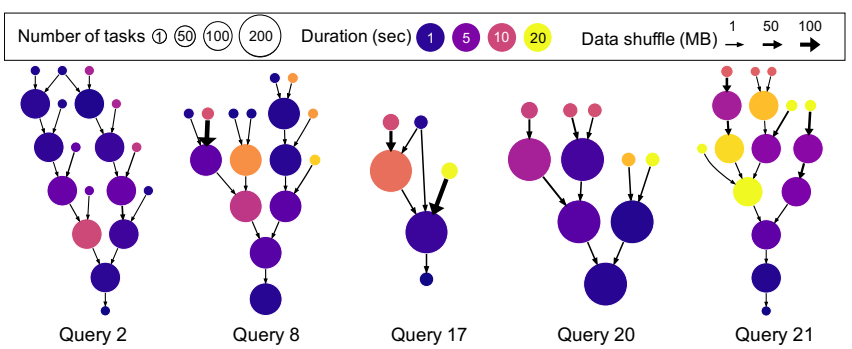

Figure 1: Data-parallel jobs have complex data-flow graphs like the ones shown (TPC-H queries in Spark), with each node having a distinct number of tasks, task durations, and input/output sizes.

For recurring jobs, which are common in production clusters [4], reasonable estimates of runtimes and intermediate data sizes may be available. Most cluster schedulers, however, ignore this job structure in their decisions and rely on e.g., coarse-grained fair sharing $[8,16$, $31,32]$, rigid priority levels [77], and manual specification of each job's parallelism [68, §5]. Existing schedulers choose to largely ignore this rich, easily-available job structure information because it is difficult to design scheduling algorithms that make use of it. We illustrate the challenges of using job-specific information in scheduling decisions with two concrete examples: $(i)$ dependency-aware scheduling, and (ii) automatically choosing the right number of parallel tasks.

\subsection{Dependency-aware task scheduling}

Many job DAGs in practice have tens or hundreds of stages with different durations and numbers of parallel tasks in a complex dependency structure. An ideal schedule ensures that independent stages run in parallel as much as possible, and that no stage ever blocks on a dependency if there are available resources. Ensuring this requires the scheduler to understand the dependency structure and plan ahead. This "DAG scheduling problem" is algorithmically hard: see, e.g., the illustrative example by Grandl et al. [36, §2.2] and the one we describe in detail in Appendix A. Theoretical research [18, 20, 48, 69] has focused mostly on simple instances of the problem that do not capture the complexity of real data processing clusters (e.g., online job arrivals, multiple DAGs, multiple tasks per stage, jobs with different inherent parallelism, overheads for moving jobs between machines, etc.). For example, in a recent paper, Agrawal et al. [5] showed that two simple DAG scheduling policies (shortest-job-first and latestarrival-processor-sharing) have constant competitive ratio in a basic model with one task per job stage. As our results show $(\S 2.3, \S 7)$, these policies are far from optimal in a real Spark cluster.

Hence, designing an algorithm to generate optimal schedules for all possible DAG combinations is intractable [36, 57]. Existing schedulers ignore this challenge: they enqueue tasks from a stage as soon as it becomes available, or run stages in an arbitrary order.

\subsection{Setting the right level of parallelism}

In addition to understanding dependencies, an ideal scheduler must also understand how to best split limited resources among jobs. Jobs vary in the amount of data that they process, and in the amount of parallel work available. A job with large input or large intermediate data can efficiently harness additional parallelism; by contrast, a job running on small input data, or one with less efficiently parallelizable operations, sees diminishing returns beyond modest parallelism. 


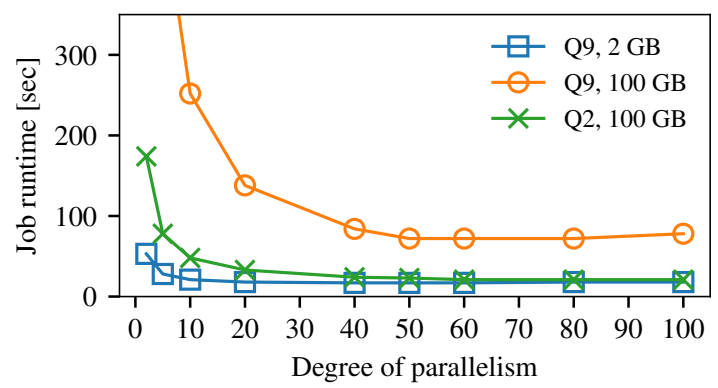

Figure 2: TPC-H queries scale differently with parallelism: Q9 on a $100 \mathrm{~GB}$ input sees speedups up to 40 parallel tasks, while Q2 stops gaining at 20 tasks; Q9 on a 2 GB input needs only 5 tasks. Picking "sweet spots" on these curves for a mixed workload is difficult.

Figure 2 illustrates this with the job runtime of two TPC-H [73] queries running on Spark as they are given additional resources to run more parallel tasks. Even when both process $100 \mathrm{~GB}$ of input, Q2 and Q9 exhibit widely different scalability: Q9 sees significant speedup up to 40 parallel tasks, while Q2 only obtains marginal returns beyond 20 tasks. When Q9 runs on a smaller input of $2 \mathrm{~GB}$, however, it needs no more than ten parallel tasks. For all jobs, assigning additional parallel tasks beyond a "sweet spot" in the curve adds only diminishing gains. Hence, the scheduler should reason about which job will see the largest marginal gain from extra resources and accordingly pick the sweet spot for each job.

Existing schedulers largely side-step this problem. Most burden the user with the choice of how many parallel tasks to use [68, §5], or rely on a separate "auto-scaling" component based on coarse heuristics $[9,28]$. Indeed, many fair schedulers [31, 43] divide resources without paying attention to their decisions' efficiency: sometimes, an "unfair" schedule results in a more efficient overall execution.

\subsection{An illustrative example on Spark}

The aspects described are just two examples of how schedulers can exploit knowledge of the workload. To achieve the best performance, schedulers must also respect other considerations, such as the execution order (e.g., favoring short jobs) and avoiding resource fragmentation $[34,77]$. Considering all these dimensions together - as Decima does - makes a substantial difference. We illustrate this by running a mix of ten randomly chosen TPC-H [73] queries with input sizes drawn from a long-tailed distribution on a Spark cluster with 50 parallel task slots. ${ }^{4}$ Figure 3 visualizes the schedules imposed by (a) Spark's default FIFO scheduling; (b) a shortest-job-first (SJF) policy that strictly prioritizes short jobs; (c) a more realistic, fair scheduler that dynamically divides task slots between jobs; and $(d)$ a scheduling policy learned by Decima. We measure average job completion time (JCT) over the ten jobs. Having access to the graph structure helps Decima improve average JCT by $45 \%$ over the naive FIFO scheduler, and by $19 \%$ over the fair scheduler. It achieves this speedup by completing short jobs quickly, as five jobs finish in the first 40 seconds; and by maximizing parallel-processing efficiency. SJF dedicates all task slots to the next-smallest job in order to finish it early (but inefficiently); by contrast, Decima runs jobs near their parallelism sweet spot. By controlling parallelism, Decima reduces

\footnotetext{
${ }^{4}$ See $\$ 7$ for details of the workload and our cluster setup.
}

the total time to complete all jobs by $30 \%$ compared to SJF. Further, unlike fair scheduling, Decima partitions task slots non-uniformly across jobs, improving average JCT by $19 \%$.

Designing general-purpose heuristics to achieve these benefits is difficult, as each additional dimension (DAG structure, parallelism, job sizes, etc.) increases complexity and introduces new edge cases. Decima opens up a new option: using data-driven techniques, it automatically learns workload-specific policies that can reap these gains. Decima does so without requiring human guidance beyond a highlevel goal (e.g., minimal average JCT), and without explicitly modeling the system or the workload.

\section{The DAG Scheduling Problem in Spark}

Decima is a general framework for learning scheduling algorithms for DAG-structured jobs. For concreteness, we describe its design in the context of the Spark system.

A Spark job consists of a DAG whose nodes are the execution stages of the job. Each stage represents an operation that the system runs in parallel over many shards of the stage's input data. The inputs are the outputs of one or more parent stages, and each shard is processed by a single task. A stage's tasks become runnable as soon as all parent stages have completed. How many tasks can run in parallel depends on the number of executors that the job holds. Usually, a stage has more tasks than there are executors, and the tasks therefore run in several "waves". Executors are assigned by the Spark master based on user requests, and by default stick to jobs until they finish. However, Spark also supports dynamic allocation of executors based on the wait time of pending tasks [9], although moving executors between jobs incurs some overhead (e.g., to tear down and launch JVMs).

Spark must therefore handle three kinds of scheduling decisions: (i) deciding how many executors to give to each job; (ii) deciding which stages' tasks to run next for each job, and (iii) deciding which task to run next when an executor becomes idle. When a stage completes, its job's DAG scheduler handles the activation of dependent child stages and enqueues their tasks with a lower-level task scheduler. The task scheduler maintains task queues from which it assigns a task every time an executor becomes idle.

We allow the scheduler to move executors between job DAGs as it sees fit (dynamic allocation). Decima focuses on DAG scheduling (i.e., which stage to run next) and executor allocation (i.e., each job's degree of parallelism). Since tasks in a stage run identical code and request identical resources, we use Spark's existing task-level scheduling.

\section{Overview and Design Challenges}

Decima represents the scheduler as an agent that uses a neural network to make decisions, henceforth referred to as the policy network. On scheduling events - e.g., a stage completion (which frees up executors), or a job arrival (which adds a DAG) — the agent takes as input the current state of the cluster and outputs a scheduling action. At a high level, the state captures the status of the DAGs in the scheduler's queue and the executors, while the actions determine which DAG stages executors work on at any given time.

Decima trains its neural network using RL through a large number of offline (simulated) experiments. In these experiments, Decima attempts to schedule a workload, observes the outcome, and provides the agent with a reward after each action. The reward is set based on Decima's high-level scheduling objective (e.g., minimize average 


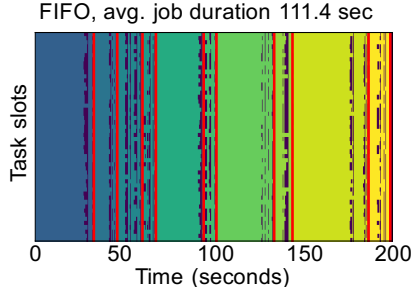

(a) FIFO scheduling.

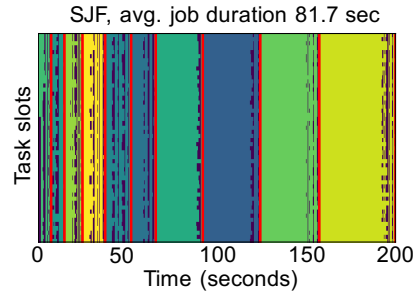

(b) SJF scheduling.

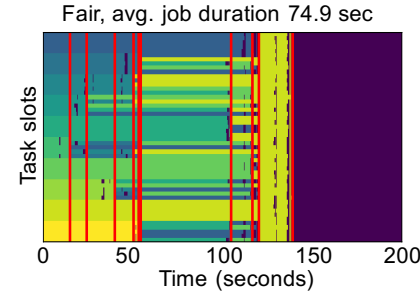

(c) Fair scheduling.

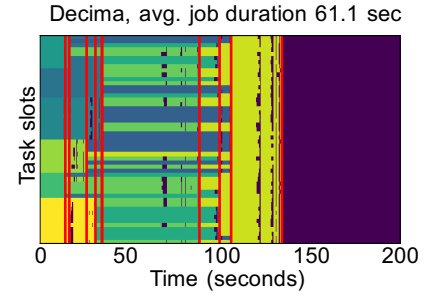

(d) Decima.

Figure 3: Decima improves average JCT of 10 random TPC-H queries by $45 \%$ over Spark's FIFO scheduler, and by $19 \%$ over a fair scheduler on a cluster with 50 task slots (executors). Different queries in different colors; vertical red lines are job completions; purple means idle.

JCT). The RL algorithm uses this reward signal to gradually improve the scheduling policy. Appendix B provides a brief primer on RL.

Decima's RL framework (Figure 4) is general and it can be applied to a variety of systems and objectives. In $\S 5$, we describe the design for scheduling DAGs on a set of identical executors to minimize average JCT. Our results in $\$ 7$ will show how to apply the same design to schedule multiple resources (e.g., CPU and memory), optimize for other objectives like makespan [65], and learn qualitatively different polices depending on the underlying system (e.g., with different overheads for moving jobs across machines).

Challenges. Decima's design tackles three key challenges:

(1) Scalable state information processing. The scheduler must consider a large amount of dynamic information to make scheduling decisions: hundreds of job DAGs, each with dozens of stages, and executors that may each be in a different state (e.g., assigned to different jobs). Processing all of this information via neural networks is challenging, particularly because neural networks usually require fixed-sized vectors as inputs.

(2) Huge space of scheduling decisions. The scheduler must map potentially thousands of runnable stages to available executors. The exponentially large space of mappings poses a challenge for RL algorithms, which must "explore" the action space in training to learn a good policy.

(3) Training for continuous stochastic job arrivals. It is important to train the scheduler to handle continuous randomly-arriving jobs over time. However, training with a continuous job arrival process is non-trivial because RL algorithms typically require training "episodes" with a finite time horizon. Further, we find that randomness in the job arrival process creates difficulties for RL training due to the variance and noise it adds to the reward.

\section{Design}

This section describes Decima's design, structured according to how it addresses the three aforementioned challenges: scalable processing of the state information ( $\$ 5.1)$, efficiently encoding scheduling decisions as actions (\$5.2), and RL training with continuous stochastic job arrivals $(\$ 5.3)$.

\subsection{Scalable state information processing}

On each state observation, Decima must convert the state information (job DAGs and executor status) into features to pass to its policy network. One option is to create a flat feature vector containing all the state information. However, this approach cannot scale to arbitrary number of DAGs of arbitrary sizes and shapes. Further, even with

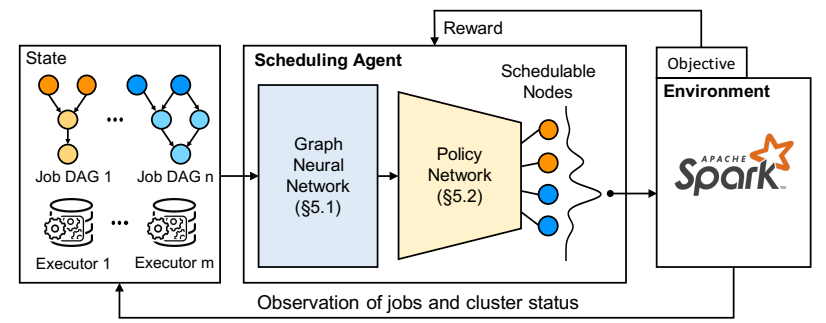

Figure 4: In Decima's RL framework, a scheduling agent observes the cluster state to decide a scheduling action on the cluster environment, and receives a reward based on a high-level objective. The agent uses a graph neural network to turn job DAGs into vectors for the policy network, which outputs actions.

\begin{tabular}{|r|l|r|l|}
\hline entity & symbol & entity & symbol \\
\hline job & $i$ & per-node feature vector & $\mathbf{x}_{v}^{i}$ \\
stage (DAG node) & $v$ & per-node embedding & $\mathbf{e}_{v}^{i}$ \\
node $v$ 's children & $\xi(v)$ & per-job embedding & $\mathbf{y}^{i}$ \\
job $i$ 's DAG & $G_{i}$ & global embedding & $\mathbf{z}$ \\
job $i$ 's parallelism & $l_{i}$ & node score & $q_{v}^{i}$ \\
non-linear functions & $f, g, q, w$ & parallelism score & $w_{l}^{i}$ \\
\hline
\end{tabular}

Table 1: Notation used throughout $\S 5$.

a hard limit on the number of jobs and stages, processing a highdimensional feature vector would require a large policy network that would be difficult to train.

Decima achieves scalability using a graph neural network, which encodes or "embeds" the state information (e.g., attributes of job stages, DAG dependency structure, etc.) in a set of embedding vectors. Our method is based on graph convolutional neural networks [12, 23, 46] but customized for scheduling. Table 1 defines our notation.

The graph embedding takes as input the job DAGs whose nodes carry a set of stage attributes (e.g., the number of remaining tasks, expected task duration, etc.), and it outputs three different types of embeddings:

(1) per-node embeddings, which capture information about the node and its children (containing, e.g., aggregated work along the critical path starting from the node);

(2) per-job embeddings, which aggregate information across an entire job DAG (containing, e.g., the total work in the job); and

(3) a global embedding, which combines information from all per-job embeddings into a cluster-level summary (containing, e.g., the number of jobs and the cluster load).

Importantly, what information to store in these embeddings is not hard-coded - Decima automatically learns what is statistically important and how to compute it from the input DAGs through endto-end training. In other words, the embeddings can be thought of as feature vectors that the graph neural network learns to compute 


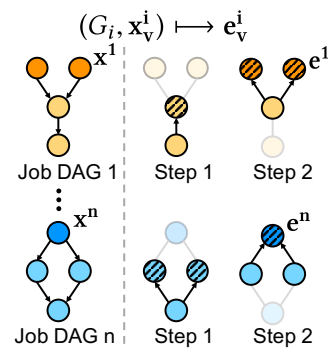

(a) Per-node embedding.

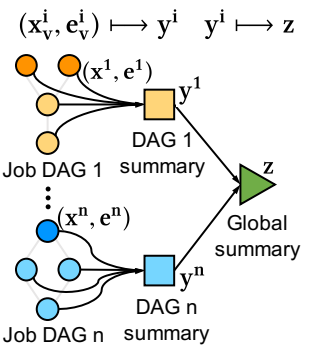

(b) Per-job and global embeddings.
Figure 5: A graph neural network transforms the raw information on each DAG node into a vector representation. This example shows two steps of local message passing and two levels of summarizations.

without manual feature engineering. Decima's graph neural network is scalable because it reuses a common set of operations as building blocks to compute the above embeddings. These building blocks are themselves implemented as small neural networks that operate on relatively low-dimensional input vectors.

Per-node embeddings. Given the vectors $\mathbf{x}_{v}^{i}$ of stage attributes corresponding to the nodes in DAG $G_{i}$, Decima builds a per-node embedding $\left(G_{i}, \mathbf{x}_{v}^{i}\right) \longmapsto \mathbf{e}_{v}^{i}$. The result $\mathbf{e}_{v}^{i}$ is a vector (e.g., in $\left.\mathbb{R}^{16}\right)$ that captures information from all nodes reachable from $v$ (i.e., $v$ 's child nodes, their children, etc.). To compute these vectors, Decima propagates information from children to parent nodes in a sequence of message passing steps, starting from the leaves of the DAG (Figure 5a). In each message passing step, a node $v$ whose children have aggregated messages from all of their children (shaded nodes in Figure 5a's examples) computes its own embedding as:

$$
\mathbf{e}_{v}^{i}=g\left[\sum_{u \in \xi(v)} f\left(\mathbf{e}_{u}^{i}\right)\right]+\mathbf{x}_{v}^{i},
$$

where $f(\cdot)$ and $g(\cdot)$ are non-linear transformations over vector inputs, implemented as (small) neural networks, and $\xi(v)$ denotes the set of $v$ 's children. The first term is a general, non-linear aggregation operation that summarizes the embeddings of $v$ 's children; adding this summary term to $v$ 's feature vector $\left(\mathbf{x}_{v}\right)$ yields the embedding for $v$. Decima reuses the same non-linear transformations $f(\cdot)$ and $g(\cdot)$ at all nodes, and in all message passing steps.

Most existing graph neural network architectures [23, 24, 46] use aggregation operations of the form $\mathbf{e}_{\mathbf{v}}=\sum_{u \in \xi(v)} f\left(\mathbf{e}_{\mathbf{u}}\right)$ to compute node embeddings. However, we found that adding a second non-linear transformation $g(\cdot)$ in Eq. (1) is critical for learning strong scheduling policies. The reason is that without $g(\cdot)$, the graph neural network cannot compute certain useful features for scheduling. For example, it cannot compute the critical path [44] of a DAG, which requires a max operation across the children of a node during message passing. ${ }^{5}$ Combining two non-linear transforms $f(\cdot)$ and $g(\cdot)$ enables Decima to express a wide variety of aggregation functions. For example, if $f$ and $g$ are identity transformations, the aggregation sums the child node embeddings; if $f \sim \log (\cdot / n), g \sim \exp (n \times \cdot)$, and $n \rightarrow \infty$, the aggregation computes the max of the child node embeddings. We show an empirical study of this embedding in Appendix E.

\footnotetext{
${ }^{5}$ The critical path from node $v$ can be computed as: $\operatorname{cp}(v)=\max _{u \in \xi(v)} \operatorname{cp}(u)+\operatorname{work}(v)$, where work $(\cdot)$ is the total work on node $v$.
}

Per-job and global embeddings. The graph neural network also computes a summary of all node embeddings for each DAG $G_{i}$, $\left\{\left(\mathbf{x}_{v}^{i}, \mathbf{e}_{v}^{i}\right), v \in G_{i}\right\} \longmapsto \mathbf{y}^{i}$; and a global summary across all DAGs, $\left\{\mathbf{y}^{1}, \mathbf{y}^{2}, \ldots\right\} \longmapsto z$. To compute these embeddings, Decima adds a summary node to each DAG, which has all the nodes in the DAG as children (the squares in Figure 5b). These DAG-level summary nodes are in turn children of a single global summary node (the triangle in Figure 5b). The embeddings for these summary nodes are also computed using Eq. (1). Each level of summarization has its own non-linear transformations $f$ and $g$; in other words, the graph neural network uses six non-linear transformations in total, two for each level of summarization.

\subsection{Encoding scheduling decisions as actions}

The key challenge for encoding scheduling decisions lies in the learning and computational complexities of dealing with large action spaces. As a naive approach, consider a solution, that given the embeddings from $\$ 5.1$, returns the assignment for all executors to job stages in one shot. This approach has to choose actions from an exponentially large set of combinations. On the other extreme, consider a solution that invokes the scheduling agent to pick one stage every time an executor becomes available. This approach has a much smaller action space $(\mathrm{O}$ (\# stages)), but it requires long sequences of actions to schedule a given set of jobs. In RL, both large action spaces and long action sequences increase sample complexity and slow down training [7, 72].

Decima balances the size of the action space and the number of actions required by decomposing scheduling decisions into a series of two-dimensional actions, which output $(i)$ a stage designated to be scheduled next, and (ii) an upper limit on the number of executors to use for that stage's job.

Scheduling events. Decima invokes the scheduling agent when the set of runnable stages - i.e., stages whose parents have completed and which have at least one waiting task - in any job DAG changes. Such scheduling events happen when (i) a stage runs out of tasks (i.e., needs no more executors), (ii) a stage completes, unlocking the tasks of one or more of its child stages, or (iii) a new job arrives to the system.

At each scheduling event, the agent schedules a group of free executors in one or more actions. Specifically, it passes the embedding vectors from $\$ 5.1$ as input to the policy network, which outputs a twodimensional action $\left\langle v, l_{i}\right\rangle$, consisting of a stage $v$ and the parallelism limit $l_{i}$ for $v$ 's job $i$. If job $i$ currently has fewer than $l_{i}$ executors, Decima assigns executors to $v$ up to the limit. If there are still free executors after the scheduling action, Decima invokes the agent again to select another stage and parallelism limit. This process repeats until all the executors have been assigned, or there are no more runnable stages. Decima ensures that this process completes in a finite number of steps by enforcing that the parallelism limit $l_{i}$ is greater than the number of executors currently allocated to job $i$, so that at least one new executor is scheduled with each action.

Stage selection. Figure 6 visualizes Decima's policy network. For a scheduling event at time $t$, during which the state is $s_{t}$, the policy network selects a stage to schedule as follows. For a node $v$ in job $i$, it computes a score $q_{v}^{i} \triangleq q\left(\mathbf{e}_{v}^{i}, \mathbf{y}^{i}, \mathbf{z}\right)$, where $q(\cdot)$ is a score function that maps the embedding vectors (output from the graph neural network in §5.1) to a scalar value. Similar to the embedding step, the score function is also a non-linear transformation implemented as a neural 


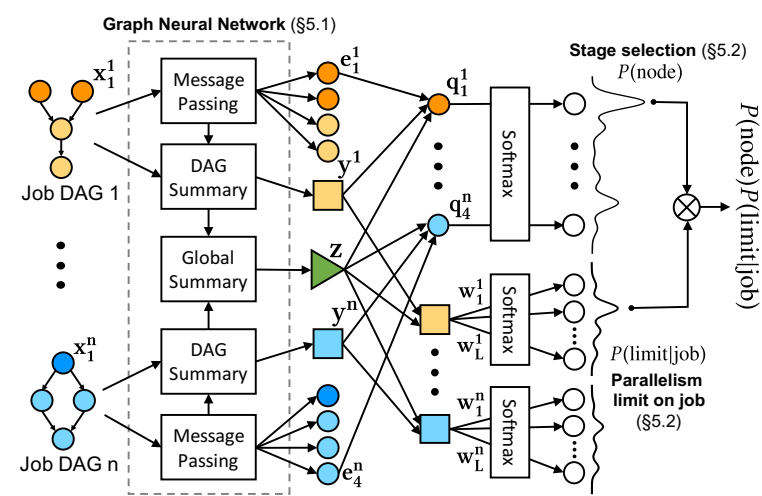

Figure 6: For each node $v$ in job $i$, the policy network uses per-node embedding $\mathrm{e}_{v}^{i}$, per-job embedding $\mathrm{y}^{i}$ and global embedding $\mathrm{z}$ to compute (i) the score $\mathrm{q}_{v}^{i}$ for sampling a node to schedule and (ii) the score $\mathrm{w}_{l}^{i}$ for sampling a parallelism limit for the node's job.

network. The score $q_{v}^{i}$ represents the priority of scheduling node $v$. Decima then uses a softmax operation [17] to compute the probability of selecting node $v$ based on the priority scores:

$$
P(\text { node }=v)=\frac{\exp \left(q_{v}^{i}\right)}{\sum_{u \in \mathcal{A}_{t}} \exp \left(q_{u}^{j(u)}\right)},
$$

where $j(u)$ is the job of node $u$, and $\mathcal{A}_{t}$ is the set of nodes that can be scheduled at time $t$. Notice that $\mathcal{A}_{t}$ is known to the RL agent at each step, since the input DAGs tell exactly which stages are runnable. Here, $\mathcal{A}_{t}$ restricts which outputs are considered by the softmax operation. The whole operation is end-to-end differentiable.

Parallelism limit selection. Many existing schedulers set a static degree of parallelism for each job: e.g., Spark by default takes the number of executors as a command-line argument on job submission. Decima adapts a job's parallelism each time it makes a scheduling decision for that job, and varies the parallelism as different stages in the job become runnable or finish execution.

For each job $i$, Decima's policy network also computes a score $w_{l}^{i} \triangleq w\left(\mathbf{y}^{i}, \mathbf{z}, l\right)$ for assigning parallelism limit $l$ to job $i$, using another score function $w(\cdot)$. Similar to stage selection, Decima applies a softmax operation on these scores to compute the probability of selecting a parallelism limit (Figure 6).

Importantly, Decima uses the same score function $w(\cdot)$ for all jobs and all parallelism limit values. This is possible because the score function takes the parallelism $l$ as one of its inputs. Without using $l$ as an input, we cannot distinguish between different parallelism limits, and would have to use separate functions for each limit. Since the number of possible limits can be as large as the number of executors, reusing the same score function significantly reduces the number of parameters in the policy network and speeds up training (Figure 15a).

Decima's action specifies job-level parallelism (e.g., ten total executors for the entire job), as opposed fine-grained stage-level parallelism. This design choice trades off granularity of control for a model that is easier to train. In particular, restricting Decima to job-level parallelism control reduces the space of scheduling policies that it must explore and optimize over during training.

However, Decima still maintains the expressivity to (indirectly) control stage-level parallelism. On each scheduling event, Decima picks a stage $v$, and new parallelism limit $l_{i}$ for $v$ 's job $i$. The system then schedules executors to $v$ until $i$ 's parallelism reaches the limit $l_{i}$. Through repeated actions with different parallelism limits, Decima can add desired numbers of executors to specific stages. For example, suppose job $i$ currently has ten executors, four of which are working on stage $v$. To add two more executors to $v$, Decima, on a scheduling event, picks stage $v$ with parallelism limit of 12 . Our experiments show that Decima achieves the same performance with job-level parallelism as with fine-grained, stage-level parallelism choice, at substantially accelerated training (Figure 15a).

\subsection{Training}

The primary challenge for training Decima is how to train with continuous stochastic job arrivals. To explain the challenge, we first describe the RL algorithms used for training.

RL training proceeds in episodes. Each episode consists of multiple scheduling events, and each scheduling event includes one or more actions. Let $T$ be the total number of actions in an episode ( $T$ can vary across different episodes), and $t_{k}$ be the wall clock time of the $k^{\text {th }}$ action. To guide the RL algorithm, Decima gives the agent a reward $r_{k}$ after each action based on its high-level scheduling objective. For example, if the objective is to minimize the average JCT, Decima penalizes the agent $r_{k}=-\left(t_{k}-t_{k-1}\right) J_{k}$ after the $k^{t h}$ action, where $J_{k}$ is the number of jobs in the system during the interval $\left[t_{k-1}, t_{k}\right)$. The goal of the RL algorithm is to minimize the expected time-average of the penalties: $\mathbb{E}\left[1 / t_{T} \sum_{k=1}^{T}\left(t_{k}-t_{k-1}\right) J_{k}\right]$. This objective minimizes the average number of jobs in the system, and hence, by Little's law $[21, \S 5]$, it effectively minimizing the average JCT.

Decima uses a policy gradient algorithm for training. The main idea in policy gradient methods is to learn by performing gradient descent on the neural network parameters using the rewards observed during training. Notice that all of Decima's operations, from the graph neural network (\$5.1) to the policy network (\$5.2), are differentiable. For conciseness, we denote all of the parameters in these operations jointly as $\theta$, and the scheduling policy as $\pi_{\theta}\left(s_{t}, a_{t}\right)$ - defined as the probability of taking action $a_{t}$ in state $s_{t}$.

Consider an episode of length $T$, where the agent collects (state, action, reward) observations, i.e., $\left(s_{k}, a_{k}, r_{k}\right)$, at each step $k$. The agent updates the parameters $\theta$ of its policy $\pi_{\theta}\left(s_{t}, a_{t}\right)$ using the REINFORCE policy gradient algorithm [79]:

$$
\theta \leftarrow \theta+\alpha \sum_{k=1}^{T} \nabla_{\theta} \log \pi_{\theta}\left(s_{k}, a_{k}\right)\left(\sum_{k^{\prime}=k}^{T} r_{k^{\prime}}-b_{k}\right) .
$$

Here, $\alpha$ is the learning rate and $b_{k}$ is a baseline used to reduce the variance of the policy gradient [78]. An example of a baseline is a "time-based" baseline [37, 53], which sets $b_{k}$ to the cumulative reward from step $k$ onwards, averaged over all training episodes. Intuitively, $\left(\sum_{k^{\prime}} r_{k^{\prime}}-b_{k}\right)$ estimates how much better (or worse) the total reward is (from step $k$ onwards) in a particular episode compared to the average case; and $\nabla_{\theta} \log \pi_{\theta}\left(s_{k}, a_{k}\right)$ provides a direction in the parameter space to increase the probability of choosing action $a_{k}$ at state $s_{k}$. As a result, the net effect of this equation is to increase the probability of choosing an action that leads to a better-than-average reward. ${ }^{6}$

\footnotetext{
${ }^{6}$ The update rule in Eq. (3) aims to maximize the sum of rewards during an episode. To maximize the time-average of the rewards, Decima uses a slightly modified form of this equation. See Appendix B for details.
} 


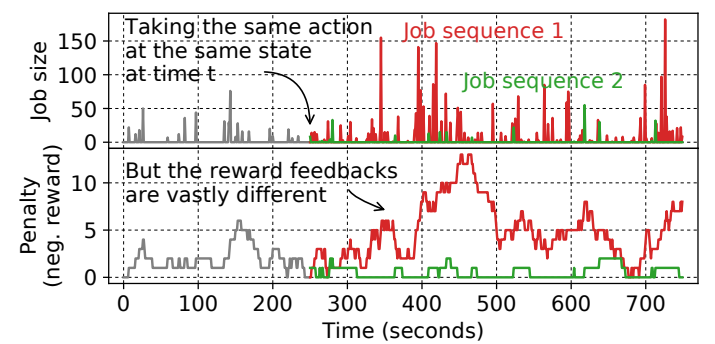

Figure 7: Illustrative example of how different job arrival sequences can lead to vastly different rewards. After time $t$, we sample two job arrival sequences, from a Poisson arrival process (10 seconds mean inter-arrival time) with randomly-sampled TPC-H queries.

Challenge \#1: Training with continuous job arrivals. To learn a robust scheduling policy, the agent has to experience "streaming" scenarios, where jobs arrive continuously over time, during training. Training with "batch" scenarios, where all jobs arrive at the beginning of an episode, leads to poor policies in streaming settings (e.g., see Figure 14). However, training with a continuous stream of job arrivals is non-trivial. In particular, the agent's initial policy is very poor (e.g., as the initial parameters are random). Therefore, the agent cannot schedule jobs as quickly as they arrive in early training episodes, and a large queue of jobs builds up in almost every episode. Letting the agent explore beyond a few steps in these early episodes wastes training time, because the overloaded cluster scenarios it encounters will not occur with a reasonable policy.

To avoid this waste, we terminate initial episodes early so that the agent can reset and quickly try again from an idle state. We gradually increase the episode length throughout the training process. Thus, initially, the agent learns to schedule short sequences of jobs. As its scheduling policy improves, we increase the episode length, making the problem more challenging. The concept of gradually increasing job sequence length — and therefore, problem complexity — during training realizes curriculum learning [14] for cluster scheduling.

One subtlety about this method is that the termination cannot be deterministic. Otherwise, the agent can learn to predict when an episode terminates, and defer scheduling certain large jobs until the termination time. This turns out to be the optimal strategy over a fixed time horizon: since the agent is not penalized for the remaining jobs at termination, it is better to strictly schedule short jobs even if it means starving some large jobs. We found that this behavior leads to indefinite starvation of some jobs at runtime (where jobs arrive indefinitely). To prevent this behavior, we use a memoryless termination process. Specifically, we terminate each training episode after a time $\tau$, drawn randomly from an exponential distribution. As explained above, the mean episode length increases during training up to a large value (e.g., a few hundreds of job arrivals on average).

Challenge \#2: Variance caused by stochastic job arrivals. Next, for a policy to generalize well in a streaming setting, the training episodes must include many different job arrival patterns. This creates a new challenge: different job arrival patterns have a large impact on performance, resulting in vastly different rewards. Consider, for example, a scheduling action at the time $t$ shown in Figure 7. If the arrival sequence following this action consists of a burst of large jobs (e.g., job sequence 1), the job queue will grow large, and the agent will incur large penalties. On the other hand, a light stream of jobs (e.g., job sequence 2) will lead to short queues and small penalties. The problem is that this difference in reward has nothing to do with the action at time $t$ - it is caused by the randomness in the job arrival process. Since the RL algorithm uses the reward to assess the goodness of the action, such variance adds noise and impedes effective training.

To resolve this problem, we build upon a recently-proposed variance reduction technique for "input-driven" environments [55], where an exogenous, stochastic input process (e.g., Decima's job arrival process) affects the dynamics of the system. The main idea is to fix the same job arrival sequence in multiple training episodes, and to compute separate baselines specifically for each arrival sequence. In particular, instead of computing the baseline $b_{k}$ in Eq. (3) by averaging over episodes with different arrival sequences, we average only over episodes with the same arrival sequence. During training, we repeat this procedure for a large number of randomly-sampled job arrival sequences ( $\$ 7.2$ and $\$ 7.3$ describe how we generate the specific datasets for training). This method removes the variance caused by the job arrival process entirely, enabling the policy gradient algorithm to assess the goodness of different actions much more accurately (see Figure 14). For the implementation details of our training and the hyperparameter settings used, see Appendix C.

\section{Implementation}

We have implemented Decima as a pluggable scheduling service that parallel data processing platforms can communicate with over an RPC interface. In $\$ 6.1$, we describe the integration of Decima with Spark. Next, we describe our Python-based training infrastructure which includes an accurate Spark cluster simulator (\$6.2).

\subsection{Spark integration}

A Spark cluster ${ }^{7}$ runs multiple parallel applications, which contain one or more jobs that together form a DAG of processing stages. The Spark master manages application execution and monitors the health of many workers, which each split their resources between multiple executors. Executors are created for, and remain associated with, a specific application, which handles its own scheduling of work to executors. Once an application completes, its executors terminate. Figure 8 illustrates this architecture.

To integrate Decima in Spark, we made two major changes:

(1) Each application's DAG scheduler contacts Decima on startup and whenever a scheduling event occurs. Decima responds with the next stage to work on and the parallelism limit (\$5.2).

(2) The Spark master contacts Decima when a new job arrives to determine how many executors to launch for it, and aids Decima by taking executors away from a job once they complete a stage.

State observations. In Decima, the feature vector $\mathbf{x}_{v}^{i}(\S 5.1)$ of a node $v$ in job DAG $i$ consists of: (i) the number of tasks remaining in the stage, (ii) the average task duration, (iii) the number of executors currently working on the node, (iv) the number of available executors, and $(v)$ whether available executors are local to the job. We picked these features by attempting to include information necessary to capture the state of the cluster (e.g., the number of executors currently assigned to each stage), as well as the statistics that may help in

\footnotetext{
${ }^{7}$ We discuss Spark's "standalone" mode of operation here (http://spark.apache.org/docs/ latest/spark-standalone.html); YARN-based deployments can, in principle, use Decima, but require modifying both Spark and YARN.
} 


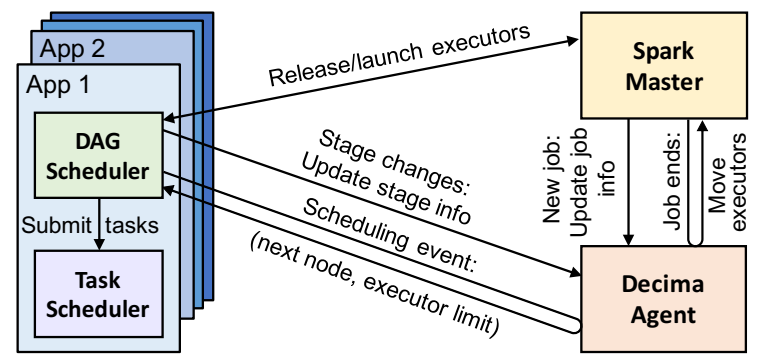

Figure 8: Spark standalone cluster architecture, with Decima additions highlighted.

scheduling decisions (e.g., a stage's average task duration). These statistics depend on the information available (e.g., profiles from past executions of the same job, or runtime metrics) and on the system used (here, Spark). Decima can easily incorporate additional signals.

Neural network architecture. The graph neural network's six transformation functions $f(\cdot)$ and $g(\cdot)(\S 5.1)$ (two each for node-level, job-level, and global embeddings) and the policy network's two score functions $q(\cdot)$ and $w(\cdot)(\S 5.2)$ are implemented using two-hiddenlayer neural networks, with 32 and 16 hidden units on each layer. Since these neural networks are reused for all jobs and all parallelism limits, Decima's model is lightweight - it consists of 12,736 parameters $(50 \mathrm{~KB})$ in total. Mapping the cluster state to a scheduling decision takes less than 15ms (Figure 15b).

\subsection{Spark simulator}

Decima's training happens offline using a faithful simulator that has access to profiling information (e.g., task durations) from a real Spark cluster $(\$ 7.2)$ and the job run time characteristics from an industrial trace (\$7.3). To faithfully simulate how Decima's decisions interact with a cluster, our simulator captures several real-world effects:

(1) The first "wave" of tasks from a particular stage often runs slower than subsequent tasks. This is due to Spark's pipelined task execution [63], JIT compilation [47] of task code, and warmup costs (e.g., making TCP connections to other executors). Decima's simulated environment thus picks the actual runtime of first-wave tasks from a different distribution than later waves.

(2) Adding an executor to a Spark job involves launching a JVM process, which takes $2-3$ seconds. Executors are tied to a job for isolation and because Spark assumes them to be long-lived. Decima's environment therefore imposes idle time reflecting the startup delay every time Decima moves an executor across jobs.

(3) A high degree of parallelism can slow down individual Spark tasks, as wider shuffles require additional TCP connections and create more work when merging data from many shards. Decima's environment captures these effects by sampling task durations from distributions collected at different levels of parallelism if this data is available.

In Appendix D, we validate the fidelity of our simulator by comparing it with real Spark executions.

\section{Evaluation}

We evaluated Decima on a real Spark cluster testbed and in simulations with a production workload from Alibaba. Our experiments address the following questions:

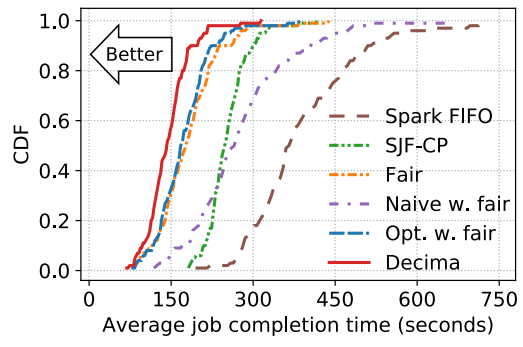

(a) Batched arrivals.

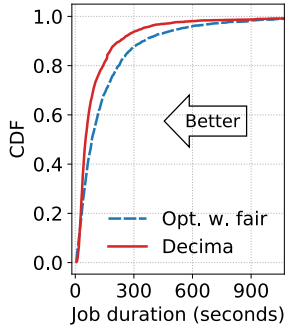

(b) Continuous arrivals
Figure 9: Decima's learned scheduling policy achieves $21 \%-3.1 \times$ lower average job completion time than baseline algorithms for batch and continuous arrivals of TPC-H jobs in a real Spark cluster.

(1) How does Decima perform compared to carefully-tuned heuristics in a real Spark cluster (\$7.2)?

(2) Can Decima's learning generalize to a multi-resource setting with different machine configurations ( $\$ 7.3)$ ?

(3) How does each of our key ideas contribute to Decima's performance; how does Decima adapt when scheduling environments change; and how fast does Decima train and make scheduling decisions after training?

\subsection{Existing baseline algorithms}

In our evaluation, we compare Decima's performance to that of seven baseline algorithms:

(1) Spark's default FIFO scheduling, which runs jobs in the same order they arrive in and grants as many executors to each job as the user requested.

(2) A shortest-job-first critical-path heuristic (SJF-CP), which prioritizes jobs based on their total work, and within each job runs tasks from the next stage on its critical path.

(3) Simple fair scheduling, which gives each job an equal fair share of the executors and round-robins over tasks from runnable stages to drain all branches concurrently.

(4) Naive weighted fair scheduling, which assigns executors to jobs proportional to their total work.

(5) A carefully-tuned weighted fair scheduling that gives each job $T_{i}^{\alpha} / \sum_{i} T_{i}^{\alpha}$ of total executors, where $T_{i}$ is the total work of each job $i$ and $\alpha$ is a tuning factor. Notice that $\alpha=0$ reduces to the simple fair scheme, and $\alpha=1$ to the naive weighted fair one. We sweep through $\alpha \in\{-2,-1.9, \ldots, 2\}$ for the optimal factor.

(6) The standard multi-resource packing algorithm from Tetris [34], which greedily schedules the stage that maximizes the dot product of the requested resource vector and the available resource vector.

(7) Graphene*, an adaptation of Graphene [36] for Decima's discrete executor classes. Graphene* detects and groups "troublesome" nodes using Graphene's algorithm [36, §4.1], and schedules them together with optimally tuned parallelism as in (5), achieving the essence of Graphene's planning strategy. We perform a grid search to optimize for the hyperparameters (details in Appendix F).

\subsection{Spark cluster}

We use an OpenStack cluster running Spark v2.2, modified as described in $§ 6.1$, in the Chameleon Cloud testbed. ${ }^{8}$ The cluster consists

\footnotetext{
${ }^{8}$ https://www.chameleoncloud.org
} 

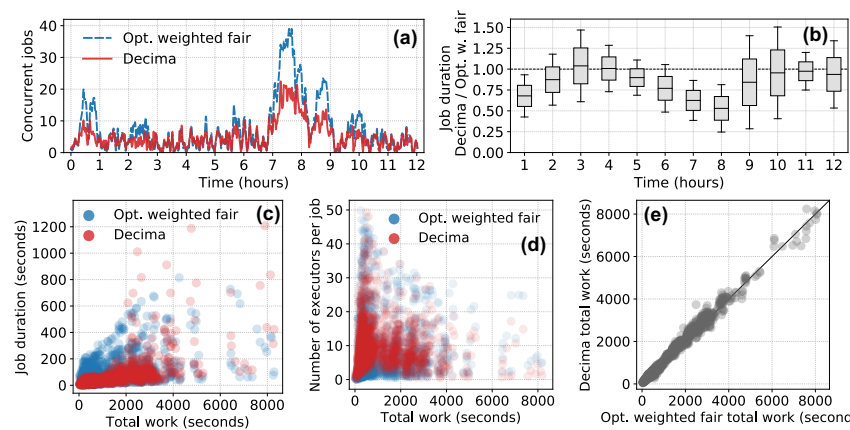

Figure 10: Time-series analysis $(\mathrm{a}, \mathrm{b})$ of continuous TPC-H job arrivals to a Spark cluster shows that Decima achieves most performance gains over heuristics during busy periods (e.g., runs jobs $2 \times$ faster during hour 8 ), as it appropriately prioritizes small jobs (c) with more executors (d), while preventing work inflation (e).

of 25 worker VMs, each running two executors on an m1.xlarge instance (8 CPUs, 16 GB RAM) and a master VM on an $\mathrm{m} 1$.xxxlarge instance (16 CPUs, 32 GB RAM). Our experiments consider ( $i$ ) batched arrivals, in which multiple jobs start at the same time and run until completion, and (ii) continuous arrivals, in which jobs arrive with stochastic interarrival distributions or follow a trace.

Batched arrivals. We randomly sample jobs from six different input sizes $(2,5,10,20,50$, and $100 \mathrm{~GB})$ and all 22 TPC-H [73] queries, producing a heavy-tailed distribution: $23 \%$ of the jobs contain $82 \%$ of the total work. A combination of 20 random jobs (unseen in training) arrives as a batch, and we measure their average JCT.

Figure 9a shows a cumulative distribution of the average JCT over 100 experiments. There are three key observations from the results. First, SJF-CP and fair scheduling, albeit simple, outperform the FIFO policy by $1.6 \times$ and $2.5 \times$ on average. Importantly, the fair scheduling policies outperform SJF-CP since they work on multiple jobs, while SJF-CP focuses all executors exclusively on the shortest job.

Second, perhaps surprisingly, unweighted fair scheduling outperforms fair scheduling weighted by job size ("naive weighted fair"). This is because weighted fair scheduling grants small jobs fewer executors than their fair share, slowing them down and increasing average JCT. Our tuned weighted fair heuristic ("opt. weighted fair") counters this effect by calibrating the weights for each job on each experiment (\$7.1). The optimal $\alpha$ is usually around -1 , i.e., the heuristic sets the number of executors inversely proportional to job size. This policy effectively focuses on small jobs early on, and later shifts to running large jobs in parallel; it outperforms fair scheduling by $11 \%$.

Finally, Decima outperforms all baseline algorithms and improves the average JCT by $21 \%$ over the closest heuristic ("opt. weighted fair"). This is because Decima prioritizes jobs better, assigns efficient executor shares to different jobs, and leverages the job DAG structure ( $\$ 7.4$ breaks down the benefit of each of these factors). Decima autonomously learns this policy through end-to-end RL training, while the best-performing baseline algorithms required careful tuning.

Continuous arrivals. We sample 1,000 TPC-H jobs of six different sizes uniformly at random, and model their arrival as a Poisson process with an average interarrival time of 45 seconds. The resulting cluster load is about $85 \%$. At this cluster load, jobs arrive faster than most heuristic-based scheduling policies can complete them. Figure $9 \mathrm{~b}$

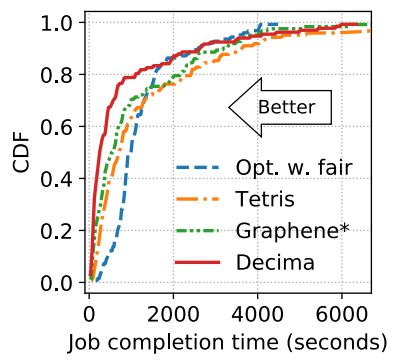

(a) Industrial trace replay.

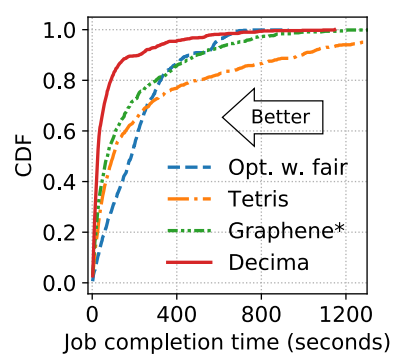

(b) TPC-H workload.

Figure 11: With multi-dimensional resources, Decima's scheduling policy outperforms Graphene* by $32 \%$ to $43 \%$ in average JCT.

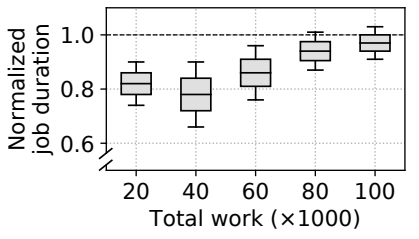

(a) Job duration grouped by to- (b) Number of executors that Decima tal work, Decima normalized to uses for "small" jobs, normalized to Graphene*.

Figure 12: Decima outperforms Graphene* with multi-dimensional resources by (a) completing small jobs faster and (b) use "oversized" executors for small jobs (smallest $20 \%$ in total work).

shows that Decima outperforms the only baseline algorithm that can keep up ("opt. weighted fair"); Decima's average JCT is $29 \%$ lower. In particular, Decima shines during busy, high-load periods, where scheduling decisions have a much larger impact than when cluster resources are abundant. Figure 10a shows that Decima maintains a lower concurrent job count than the tuned heuristic particularly during the busy period in hours 7-9, where Decima completes jobs about $2 \times$ faster (Figure 10b). Performance under high load is important for batch processing clusters, which often have long job queues [66], and periods of high load are when good scheduling decisions have the most impact (e.g., reducing the overprovisioning required for workload peaks).

Decima's performance gain comes from finishing small jobs faster, as the concentration of red points in the lower-left corner of Figure 10c shows. Decima achieves this by assigning more executors to the small jobs (Figure 10d). The right number of executors for each job is workload-dependent: indiscriminately giving small jobs more executors would use cluster resources inefficiently (\$2.2). For example, SJF-CP's strictly gives all available executors to the smallest job, but this inefficient use of executors inflates total work, and SJF-CP therefore accumulates a growing backlog of jobs. Decima's executor assignment, by contrast, results in similar total work as with the hand-tuned heuristic. Figure 10e shows this: jobs below the diagonal have smaller total work with Decima than with the heuristic, and ones above have larger total work in Decima. Most small jobs are on the diagonal, indicating that Decima only increases the parallelism limit when extra executors are still efficient. Consequently, Decima successfully balances between giving small jobs extra resources to finish them sooner and using the resources efficiently. 


\subsection{Multi-dimensional resource packing}

The standalone Spark scheduler used in our previous experiments only provides jobs with access to predefined executor slots. More advanced cluster schedulers, such as YARN [75] or Mesos [41], allow jobs to specify their tasks' resource requirements and create appropriately-sized executors. Packing tasks with multi-dimensional resource needs (e.g., 〈CPU, memory $\rangle$ ) onto fixed-capacity servers adds further complexity to the scheduling problem [34, 36]. We use a production trace from Alibaba to investigate if Decima can learn good multi-dimensional scheduling policies with the same core approach.

Industrial trace. The trace contains about 20,000 jobs from a production cluster. Many jobs have complex DAGs: $59 \%$ have four or more stages, and some have hundreds. We run the experiments using our simulator ( $\$ 6.2)$ with up to 30,000 executors. This parameter is set according to the maximum number of concurrent tasks in the trace. We use the first half of the trace for training and then compare Decima's performance with other schemes on the remaining portion.

Multi-resource environment. We modify Decima's environment to provide several discrete executor classes with different memory sizes. Tasks now require a minimum amount of CPU and memory, i.e., a task must fit into the executor that runs it. Tasks can run in executors larger than or equal to their resource request. Decima now chooses a DAG stage to schedule, a parallelism level, and an executor class to use. Our experiments use four executor types, each with 1 CPU core and $(0.25,0.5,0.75,1)$ unit of normalized memory; each executor class makes up $25 \%$ of total cluster executors.

Results. We run simulated multi-resource experiments on continuous job arrivals according to the trace. Figure 11a shows the results for Decima and three other algorithms: the optimally tuned weighted-fair heuristic, Tetris, and Graphene*. Decima achieves a 32\% lower average JCT than the best competing algorithm (Graphene*), suggesting that it learns a good policy in the multi-resource environment.

Decima's policy is qualitatively different to Graphene*'s. Figure 12a breaks Decima's improvement over Graphene* down by jobs' total work. Decima completes jobs faster than Graphene* for all job sizes, but its gain is particularly large for small jobs. The reason is that Decima learns to use "oversized" executors when they can help finish nearly-completed small jobs when insufficiently many right-sized executors are available. Figure 12b illustrates this: Decima uses 39\% more executors of the largest class on the jobs with smallest $20 \%$ total work (full profiles in Appendix G). In other words, Decima trades off memory fragmentation against clearing the job queue more quickly. This trade-off makes sense because small jobs $(i)$ contribute more to the average JCT objective, and (ii) only fragment resources for a short time. By contrast, Tetris greedily packs tasks into the bestfitting executor class and achieves the lowest memory fragmentation. Decima's fragmentation is within $4 \%-13 \%$ of Tetris's, but Decima's average JCT is $52 \%$ lower, as it learns to balance the trade-off well. This requires respecting workload-dependent factors, such as the DAG structure, the threshold for what is a "small" job, and others. Heuristic approaches like Graphene* attempt to balance those factors via additive score functions and extensive tuning, while Decima learns them without such inputs.

We also repeat this experiment with the TPC-H workload, using 200 executors and sampling each TPC-H DAG node's memory request from $(0,1]$. Figure $11 \mathrm{~b}$ shows that Decima outperforms the competing algorithms by even larger margins (e.g., 43\% over Graphene*). This is because the industrial trace lacks work inflation measurements for different levels of parallelism, which we provide for TPC-H. Decima learns to use this information to further calibrate executor assignment.

\subsection{Decima deep dive}

Finally, we demonstrate the wide range of scheduling policies Decima can learn, and break down the impact of our key ideas and techniques on Decima's performance. In appendices, we further evaluate Decima's optimality via an exhaustive search of job orderings (Appendix $\mathrm{H}$ ), the robustness of its learned policies to changing environments (Appendix I), and Decima's sensitivity to incomplete information (Appendix J).

Learned policies. Decima outperforms other algorithms because it can learn different policies depending on the high-level objective, the workload, and environmental conditions. When Decima optimizes for average JCT (Figure 13a), it learns to share executors for small jobs to finish them quickly and avoids inefficiently using too many executors on large jobs (\$7.2). Decima also keeps the executors working on tasks from the same job to avoid the overhead of moving executors (§6.1). However, if moving executors between jobs is free - as is effectively the case for long tasks, or for systems without JVM spawn overhead - Decima learns a policy that eagerly moves executors among jobs (cf. the frequent color changes in Figure 13b). Finally, given a different objective of minimizing the overall makespan for a batch of jobs, Decima learns yet another different policy (Figure 13c). Since only the final job's completion time matters for a makespan objective, Decima no longer works to finish jobs early. Instead, many jobs complete together at the end of the batched workload, which gives the scheduler more choices of jobs throughout the execution, increasing cluster utilization.

Impact of learning architecture. We validate that Decima uses all raw information provided in the state and requires all its key design components by selectively omitting components. We run 1,000 continuous TPC-H job arrivals on a simulated cluster at different loads, and train five different variants of Decima on each load.

Figure 14 shows that removing any one component from Decima results in worse average JCTs than the tuned weighted-fair heuristic at a high cluster load. There are four takeaways from this result. First, parallelism control has the greatest impact on Decima's performance. Without parallelism control, Decima assigns all available executors to a single stage at every scheduling event. Even at a moderate cluster load (e.g., 55\%), this leads to an unstable policy that cannot keep up with the arrival rate of incoming jobs. Second, omitting the graph embedding (i.e., directly taking raw features on each node as input to the score functions in \$5.2) makes Decima unable to estimate remaining work in a job and to account for other jobs in the cluster. Consequently, Decima has no notion of small jobs or cluster load, and its learned policy quickly becomes unstable as the load increases. Third, using unfixed job sequences across training episodes increases the variance in the reward signal (\$5.3). As the load increases, job arrival sequences become more varied, which increases variance in the reward. At cluster load larger than $75 \%$, reducing this variance via synchronized termination improves average JCT by $2 \times$ when training Decima, illustrating that variance reduction is key to learning high-quality policies 


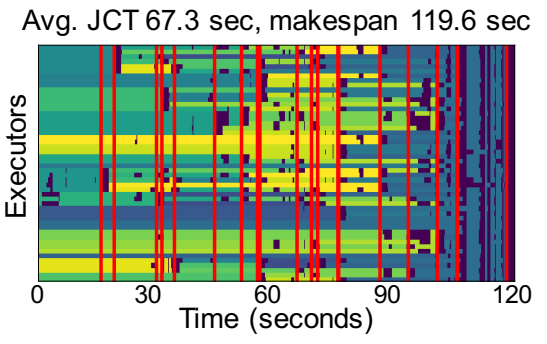

(a) Average JCT objective.

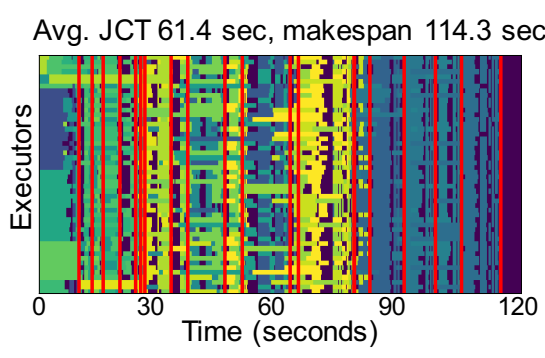

(b) Avg. JCT, with zero-cost executor motion.

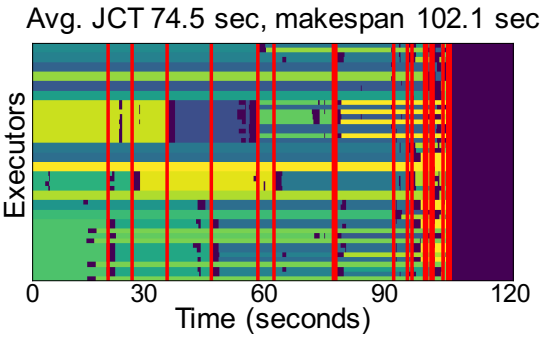

(c) Minimal makespan objective.

Figure 13: Decima learns qualitatively different policies depending on the environment (e.g., costly (a) vs. free executor migration (b)) and the objective (e.g., average JCT (a) vs. makespan (c)). Vertical red lines indicate job completions, colors indicate tasks in different jobs, and dark purple is idle time.

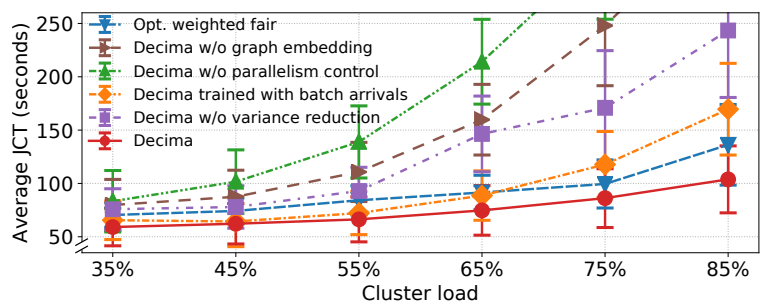

Figure 14: Breakdown of each key idea's contribution to Decima with continuous job arrivals. Omitting any concept increases Decima's average JCT above that of the weighted fair policy.

\begin{tabular}{|r|l|}
\hline Setup (IAT: interarrival time) & Average JCT [sec] \\
\hline Opt. weighted fair (best heuristic) & $91.2 \pm 23.5$ \\
\hline Decima, trained on test workload (IAT: 45 sec) & $65.4 \pm 28.7$ \\
Decima, trained on anti-skewed workload (IAT: 75 sec) & $104.8 \pm 37.6$ \\
Decima, trained on mixed workloads & $82.3 \pm 31.2$ \\
Decima, trained on mixed workloads with interarrival time hints & $76.6 \pm 33.4$ \\
\hline
\end{tabular}

Table 2: Decima generalizes to changing workloads. For an unseen workload, Decima outperforms the best heuristic by $10 \%$ when trained with a mix of workloads; and by $16 \%$ if it knows the interarrival time from an input feature.

in long-horizon scheduling problems. Fourth, training only on batched job arrivals cannot generalize to continuous job arrivals. When trained on batched arrivals, Decima learns to systematically defer large jobs, as this results in the lowest sum of JCTs (lowest sum of penalties). With continuous job arrivals, this policy starves large jobs indefinitely as the cluster load increases and jobs arrive more frequently. Consequently, Decima underperforms the tuned weighted-fair heuristic at loads above $65 \%$ when trained on batched arrivals.

Generalizing to different workloads. We test Decima's ability to generalize by changing the training workload in the TPC-H experiment (\$7.2). To simulate shifts in cluster workload, we train models for different job interarrival times between 42 and 75 seconds, and test them using a workload with a 45 second interarrival time. As Decima learns workload-specific policies, we expect its effectiveness to depend on whether broad test workload characteristics, such as interarrival time and job size distributions, match the training workload.

Table 2 shows the resulting average JCT. Decima performs well when trained on a workload similar to the test workload. Unsurprisingly, when Decima trains with an "anti-skewed" workload (75 seconds interarrival time), it generalizes poorly and underperforms the optimized weighted fair policy. This makes sense because Decima incorporates the learned interarrival time distribution in its policy.

When training with a mixed set of workloads that cover the whole interarrival time range, Decima can learn a more general policy. This

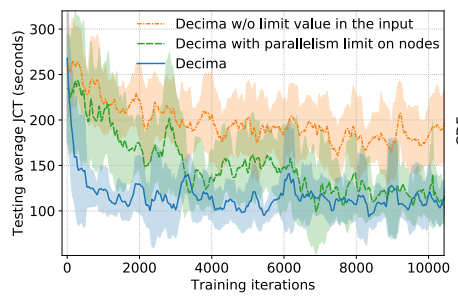

(a) Learning curve.

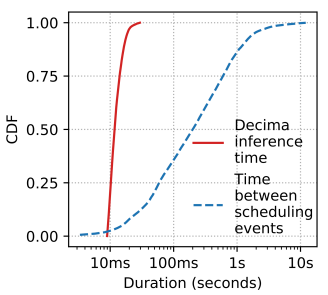

(b) Scheduling delay.
Figure 15: Different encodings of jobs parallelism (§5.2) affect Decima’s training time. Decima makes low-latency scheduling decisions: on average, the latency is about $50 \times$ smaller than the interval between scheduling events.

policy fits less strongly to a specific interarrival time distribution and therefore becomes more robust to workload changes. If Decima can observe the interarrival time as a feature in its state (\$6.1), it generalizes better still and learns an adaptive policy that achieves a $16 \%$ lower average JCT than the best heuristic. These results highlight that a diverse training workload set helps make Decima's learned policies robust to workload shifts; we discuss possible online learning in $§ 8$.

Training and inference performance. Figure 15a shows Decima's learning curve (in blue) on continuous TPC-H job arrivals (\$7.2), testing snapshots of the model every 100 iterations on (unseen) job arrival sequences. Each training iteration takes about 5 seconds. Decima’s design ( $\$ 5.3)$ is crucial for training efficiency: omitting the parallelism limit values in the input (yellow curve) forces Decima to use separate score functions for different limits, significantly increasing the number of parameters to optimize over; putting fine-grained parallelism control on nodes (green curve) slows down training as it increases the space of algorithms Decima must explore.

Figure $15 \mathrm{~b}$ shows cumulative distributions of the time Decima takes to decide on a scheduling action (in red) and the time interval between scheduling events (in blue) in our Spark testbed (\$7.2). The average scheduling delay for Decima is less than $15 \mathrm{~ms}$, while the interval between scheduling events is typically in the scale of seconds. In less than $5 \%$ of the cases, the scheduling interval is shorter than the scheduling delay (e.g., when the cluster requests for multiple scheduling actions in a single scheduling event). Thus Decima's scheduling delay imposes no measurable overhead on task runtimes.

\section{Discussion}

In this section, we discuss future research directions and other potential applications for Decima's techniques. 
Robustness and generalization. Our experiments in $\$ 7.4$ showed that Decima can learn generalizable scheduling policies that work well on an unseen workload. However, more drastic workload changes than interarrival time shifts could occur. To increase robustness of a scheduling policy against such changes, it may be helpful to train the agent on worst-case situations or adversarial workloads, drawing on the emerging literature on robust adversarial RL [64]. Another direction is to adjust the scheduling policy online as the workload changes. The key challenge with an online approach is to reduce the large sample complexity of model-free RL when the workload changes quickly. One viable approach might be to use meta learning [22, 27, 29], which allows training a "meta" scheduling agent that is designed to adapt to a specific workload with only a few observations.

Other learning objectives. In our experiments, we evaluated Decima on metrics related to job duration (e.g., average JCT, makespan). Shaping the reward signal differently can steer Decima to meet other objectives, too. For example, imposing a hard penalty whenever the deadline of a job is missed would guide Decima to a deadline-aware policy. Alternatively, basing the reward on e.g., the 90th percentile of empirical job duration samples, Decima can optimize for a tight tail of the JCT distribution. Addressing objectives formulated as constrained optimization (e.g., to minimize average JCT, but strictly guarantee fairness) using RL is an interesting further direction [2,30].

Preemptive scheduling. Decima currently never preempts running tasks and can only remove executors from a job after a stage completes. This design choice keeps the MDP tractable for RL and results in effective learning and strong scheduling policies. However, future work might investigate more fine-grained and reactive preemption in an RL-driven scheduler such as Decima. Directly introducing preemption would lead to a much larger action space (e.g., specifying arbitrary set of executors to preempt) and might require a much higher decision-making frequency. To make the RL problem tractable, one potential research direction is to leverage multi-agent $R L$ [38, 50, 62]. For example, a Decima-like scheduling agent might controls which stage to run next and how many executors to assign, and, concurrently, another agent might decide where to preempt executors.

Potential networking and system applications. Some techniques we developed for Decima are broadly applicable to other networking and computer systems problems. For example, the scalable representation of input DAGs (\$5.1) has applications in problems over graphs, such as database query optimization [56] and hardware device placement [3]. Our variance reduction technique (\$5.3) generally applies to systems with stochastic, unpredictable inputs $[54,55]$.

\section{Related Work}

There is little prior work on applying machine learning techniques to cluster scheduling. DeepRM [53], which uses RL to train a neural network for multi-dimensional resource packing, is closest to Decima in aim and approach. However, DeepRM only deals with a basic setting in which each job is a single task and was evaluated in simple, simulated environments. DeepRM's learning model also lacks support for DAG-structured jobs, and its training procedure cannot handle realistic cluster workloads with continuous job arrivals. In other applications, Mirhoseini et al.'s work on learning device placement in TensorFlow (TF) computations [60] also uses RL, but relies on recurrent neural networks to scan through all nodes for state embedding, rather than a graph neural network. Their approach use recurrent neural networks to scan through all nodes for state embedding instead of using a scalable graph neural network. The objective there is to schedule a single TF job well, and the model cannot generalize to unseen job combinations [59].

Prior work in machine learning and algorithm design has combined RL and graph neural networks to optimize complex combinatorial problems, such as vertex set cover and the traveling salesman problem $[23,49]$. The design of Decima's scalable state representation is inspired by this line of work, but we found that off-the-shelf graph neural networks perform poorly for our problem. To train strong scheduling agents, we had to change the graph neural network architecture to enable Decima to compute, amongst other metrics, the critical path of a DAG ( $\$ 5.1)$.

For resource management systems more broadly, Paragon [25] and Quasar [26] use collaborative filtering to match workloads to different machine types and avoid interference; their goal is complementary to Decima's. Tetrisched [74], like Decima, plans ahead in time, but uses a constraint solver to optimize job placement and requires the user to supply explicit constraints with their jobs. Firmament [33] also uses a constraint solver and achieves high-quality placements, but requires an administrator to configure an intricate scheduling policy. Graphene [36] uses heuristics to schedule job DAGs, but cannot set appropriate parallelism levels. Some systems "auto-scale" parallelism levels to meet job deadlines [28] or opportunistically accelerate jobs using spare resources $[68, \S 5]$. Carbyne [35] allows jobs to "altruistically" give up some of their short-term fair share of cluster resources in order to improve JCT across jobs while guarantee long-term fairness. Decima learns policies similar to Carbyne's, balancing resource shares and packing for low average JCT, but the current design of Decima does not have fairness an objective.

General-purpose cluster managers like Borg [77], Mesos [41], or YARN [75] support many different applications, making workloadspecific scheduling policies are difficult to apply at this level. However, Decima could run as a framework atop Mesos or Omega [68].

\section{Conclusion}

Decima demonstrates that automatically learning complex cluster scheduling policies using reinforcement learning is feasible, and that the learned policies are flexible and efficient. Decima's learning innovations, such as its graph embedding technique and the training framework for streaming, may be applicable to other systems processing DAGs (e.g., query optimizers). We will open source Decima, our models, and our experimental infrastructure at https://web.mit.edu/decima. This work does not raise any ethical issues.

Acknowledgments. We thank our shepherd, Aditya Akella, and the SIGCOMM reviewers for their valuable feedback. We also thank Akshay Narayan, Amy Ousterhout, Prateesh Goyal, Peter Iannucci, and Songtao He for fruitful discussions throughout the development of this project. We appreciate Haiyang Ding and Yihui Feng from Alibaba Cloud Intelligence for sharing the production cluster dataset. This work was funded in part by the NSF grants CNS-1751009, CNS-1617702, a Google Faculty Research Award, an AWS Machine Learning Research Award, a Cisco Research Center Award, an Alfred P. Sloan Research Fellowship, and sponsors of the MIT DSAIL lab. 


\section{References}

[1] Martín Abadi, Paul Barham, Jianmin Chen, Zhifeng Chen, Andy Davis, Jeffrey Dean, Matthieu Devin, Sanjay Ghemawat, Geoffrey Irving, Michael Isard, Manjunath Kudlur, Josh Levenberg, Rajat Monga, Sherry Moore, Derek G. Murray, Benoit Steiner, Paul Tucker, Vijay Vasudevan, Pete Warden, Martin Wicke, Yuan Yu, and Xiaoqiang Zheng. 2016. TensorFlow: A System for Large-scale Machine Learning. In Proceedings of the $12^{\text {th }}$ USENIX Conference on Operating Systems Design and Implementation (OSDI). 265-283. http://dl.acm.org/citation.cfm?id=3026877.3026899

[2] Joshua Achiam, David Held, Aviv Tamar, and Pieter Abbeel. 2017. Constrained policy optimization. In Proceedings of the $34^{\text {th }}$ International Conference on Machine Learning-Volume 70. 22-31.

[3] Ravichandra Addanki, Shaileshh Bojja Venkatakrishnan, Shreyan Gupta, Hongzi Mao, and Mohammad Alizadeh. 2018. Placeto: Efficient Progressive Device Placement Optimization. In Proceedings of the $1^{\text {st }}$ Machine Learning for Systems Workshop.

[4] Sameer Agarwal, Srikanth Kandula, Nicolas Bruno, Ming-Chuan Wu, Ion Stoica, and Jingren Zhou. 2012. Re-optimizing Data-parallel Computing. In Proceedings of the $9^{\text {th }}$ USENIX Conference on Networked Systems Design and Implementation (NSDI). 281-294. http://dl.acm.org/citation.cfm?id=2228298.2228327

[5] Kunal Agrawal, Jing Li, Kefu Lu, and Benjamin Moseley. 2016. Scheduling parallel DAG jobs online to minimize average flow time. In Proceedings of the $27^{\text {th }}$ annual ACM-SIAM symposium on Discrete Algorithms (SODA). Society for Industrial and Applied Mathematics, 176-189.

[6] Alibaba. 2017. Cluster data collected from production clusters in Alibaba for cluster management research. https://github.com/alibaba/clusterdata. (2017).

[7] Dario Amodei and Danny Hernandez. 2018. AI and Compute. https://openai.com/blog/ai-and-compute/. (2018).

[8] Apache Hadoop. 2014. Hadoop Fair Scheduler. (2014). http: //hadoop.apache.org/common/docs/stable1/fair_scheduler.html

[9] Apache Spark. 2018. Spark: Dynamic Resource Allocation. (2018). http //spark.apache.org/docs/2.2.1/job-scheduling.html\#dynamic-resource-allocation Spark v2.2.1 Documentation.

[10] Apache Tez 2013. Apache Tez Project. https://tez.apache.org/. (2013).

[11] Luiz André Barroso, Jimmy Clidaras, and Urs Hölzle. 2013. The Datacenter as a Computer: An Introduction to the Design of Warehouse-Scale Machines, second edition. Synthesis Lectures on Computer Architecture 8, 3 (July 2013). https://doi.org/10.2200/S00516ED2V01Y201306CAC024

[12] Peter W. Battaglia, Jessica B. Hamrick, Victor Bapst, Alvaro Sanchez-Gonzalez, Vinícius Flores Zambaldi, Mateusz Malinowski, Andrea Tacchetti, David Raposo, Adam Santoro, Ryan Faulkner, Çaglar Gülçehre, Francis Song, Andrew J. Ballard, Justin Gilmer, George E. Dahl, Ashish Vaswani, Kelsey Allen, Charles Nash, Victoria Langston, Chris Dyer, Nicolas Heess, Daan Wierstra, Pushmeet Kohli, Matthew Botvinick, Oriol Vinyals, Yujia Li, and Razvan Pascanu. 2018. Relational inductive biases, deep learning, and graph networks. arXiv preprint arXiv:1806.01261 (2018).

[13] Richard Bellman. 1966. Dynamic programming. Science 153, 3731 (1966), 34-37.

[14] Yoshua Bengio, Jérôme Louradour, Ronan Collobert, and Jason Weston. 2009. Curriculum learning. In Proceedings of the $26^{\text {th }}$ annual International Conference on Machine Learning (ICML). 41-48.

[15] Dimitri P Bertsekas and John N Tsitsiklis. 1995. Neuro-dynamic programming an overview. In Decision and Control, 1995., Proceedings of the 34th IEEE Conference on, Vol. 1. IEEE, 560-564.

[16] Arka A. Bhattacharya, David Culler, Eric Friedman, Ali Ghodsi, Scott Shenker, and Ion Stoica. 2013. Hierarchical Scheduling for Diverse Datacenter Workloads. In Proceedings of the $4^{\text {th }}$ Annual Symposium on Cloud Computing (SoCC). Article 4, 15 pages. https://doi.org/10.1145/2523616.2523637

[17] Christopher M. Bishop. 2006. Pattern Recognition and Machine Learning. Springer.

[18] Robert D Blumofe and Charles E Leiserson. 1999. Scheduling multithreaded computations by work stealing. Journal of the ACM (JACM) 46, 5 (1999), 720-748.

[19] Craig Chambers, Ashish Raniwala, Frances Perry, Stephen Adams, Robert R. Henry, Robert Bradshaw, and Nathan Weizenbaum. 2010. FlumeJava: Easy, Efficient Data-parallel Pipelines. In Proceedings of the 2010 ACM SIGPLAN Conference on Programming Language Design and Implementation (PLDI). 363-375. https://doi.org/10.1145/1806596.1806638

[20] Chandra Chekuri, Ashish Goel, Sanjeev Khanna, and Amit Kumar. 2004. Multiprocessor scheduling to minimize flow time with $\varepsilon$ resource augmentation. In Proceedings of the $36^{\text {th }}$ Annual ACM Symposium on Theory of Computing. 363-372.

[21] Dilip Chhajed and Timothy J Lowe. 2008. Building intuition: insights from basic operations management models and principles. Vol. 115. Springer Science \& Business Media.

[22] Ignasi Clavera, Jonas Rothfuss, John Schulman, Yasuhiro Fujita, Tamim Asfour, and Pieter Abbeel. 2018. Model-based reinforcement learning via meta-policy optimization. arXiv preprint arXiv: 1809.05214 (2018).
[23] Hanjun Dai, Elias B. Khalil, Yuyu Zhang, Bistra Dilkina, and Le Song. 2017. Learning Combinatorial Optimization Algorithms over Graphs. In Proceedings of the $31^{\text {st }}$ Conference on Neural Information Processing Systems (NeurIPS). 6348-6358.

[24] Michaël Defferrard, Xavier Bresson, and Pierre Vandergheynst. 2016. Convolutional Neural Networks on Graphs with Fast Localized Spectral Filtering. arXiv preprint arXiv:1606.09375 (2016)

[25] Christina Delimitrou and Christos Kozyrakis. 2013. Paragon: QoS-aware Scheduling for Heterogeneous Datacenters. In Proceedings of the $18^{\text {th }}$ International Conference on Architectural Support for Programming Languages and Operating Systems (ASPLOS). 77-88. https://doi.org/10.1145/2451116.2451125

[26] Christina Delimitrou and Christos Kozyrakis. 2014. Quasar: Resource-efficient and QoS-aware Cluster Management. In Proceedings of the $19^{\text {th }}$ International Conference on Architectural Support for Programming Languages and Operating Systems (ASPLOS). 127-144. https://doi.org/10.1145/2541940.2541941

[27] Yan Duan, John Schulman, Xi Chen, Peter L Bartlett, Ilya Sutskever, and Pieter Abbeel. 2016. RL2: Fast Reinforcement Learning via Slow Reinforcement Learning. arXiv preprint arXiv:1611.02779 (2016).

[28] Andrew D Ferguson, Peter Bodik, Srikanth Kandula, Eric Boutin, and Rodrigo Fonseca. 2012. Jockey: guaranteed job latency in data parallel clusters. In Proceedings of the $7^{\text {th }}$ ACM European Conference on Computer Systems (EuroSys).

[29] Chelsea Finn, Pieter Abbeel, and Sergey Levine. 2017. Model-Agnostic Meta-Learning for Fast Adaptation of Deep Networks. In Proceedings of the $34^{\text {th }}$ International Conference on Machine Learning (ICML). 1126-1135.

[30] Peter Geibel. 2006. Reinforcement learning for MDPs with constraints. In Proceedings of the $17^{\text {th }}$ European Conference on Machine Learning (ECML). 646-653.

[31] Ali Ghodsi, Matei Zaharia, Benjamin Hindman, Andy Konwinski, Scott Shenker, and Ion Stoica. 2011. Dominant Resource Fairness: Fair Allocation of Multiple Resource Types. In Proceedings of the $8^{\text {th }}$ USENIX Symposium on Networked Systems Design and Implementation (NSDI). 323-336. http://dl.acm.org/citation.cfm?id=1972457.1972490

[32] Ali Ghodsi, Matei Zaharia, Scott Shenker, and Ion Stoica. 2013. Choosy: max-min fair sharing for datacenter jobs with constraints. In Proceedings of the $8^{\text {th }}$ ACM European Conference on Computer Systems (EuroSys). 365-378. https://doi.org/10.1145/2465351.2465387

[33] Ionel Gog, Malte Schwarzkopf, Adam Gleave, Robert N. M. Watson, and Steven Hand. 2016. Firmament: fast, centralized cluster scheduling at scale. In Proceedings of the $12^{\text {th }}$ USENIX Symposium on Operating Systems Design and Implementation (OSDI). 99-115.

[34] Robert Grandl, Ganesh Ananthanarayanan, Srikanth Kandula, Sriram Rao, and Aditya Akella. 2014. Multi-resource Packing for Cluster Schedulers. In Proceedings of the 2014 ACM SIGCOMM Conference (SIGCOMM). 455-466.

[35] Robert Grandl, Mosharaf Chowdhury, Aditya Akella, and Ganesh Ananthanarayanan. 2016. Altruistic Scheduling in Multi-resource Clusters. In Proceedings of the $12^{\text {th }}$ USENIX Conference on Operating Systems Design and Implementation (OSDI). 65-80. http://dl.acm.org/citation.cfm?id=3026877.3026884

[36] Robert Grandl, Srikanth Kandula, Sriram Rao, Aditya Akella, and Janardhan Kulkarni. 2016. Graphene: Packing and dependency-aware scheduling for data-parallel clusters. In Proceedings of the $12^{\text {th }}$ USENIX Symposium on Operating Systems Design and Implementation (OSDI). 81-97.

[37] Evan Greensmith, Peter L Bartlett, and Jonathan Baxter. 2004. Variance reduction techniques for gradient estimates in reinforcement learning. Journal of Machine Learning Research 5, Nov (2004), 1471-1530.

[38] Jayesh K Gupta, Maxim Egorov, and Mykel Kochenderfer. 2017. Cooperative multi-agent control using deep reinforcement learning. In Proceedings of the 2017 International Conference on Autonomous Agents and Multiagent Systems (AAMAS). 66-83.

[39] Martin T Hagan, Howard B Demuth, Mark H Beale, and Orlando De Jesús. 1996. Neural network design. PWS publishing company Boston.

[40] W Keith Hastings. 1970. Monte Carlo sampling methods using Markov chains and their applications. Biometrika 1 (1970).

[41] Benjamin Hindman, Andy Konwinski, Matei Zaharia, Ali Ghodsi, Anthony D Joseph, Randy H Katz, Scott Shenker, and Ion Stoica. 2011. Mesos: A Platform for Fine-Grained Resource Sharing in the Data Center. In Proceedings of the $8^{\text {th }}$ USENIX Conference on Networked Systems Design and Implementation (NSDI).

[42] Michael Isard, Mihai Budiu, Yuan Yu, Andrew Birrell, and Dennis Fetterly. 2007. Dryad: Distributed Data-parallel Programs from Sequential Building Blocks. In Proceedings of the $2^{\text {nd }}$ ACM SIGOPS/EuroSys European Conference on Computer Systems (EuroSys). 59-72. https://doi.org/10.1145/1272996.1273005

[43] Michael Isard, Vijayan Prabhakaran, Jon Currey, Udi Wieder, Kunal Talwar, and Andrew Goldberg. 2009. Quincy: fair scheduling for distributed computing clusters. In Proceedings of the $22^{\text {nd }}$ ACM Symposium on Operating Systems Principles (SOSP). 261-276. https://doi.org/10.1145/1629575.1629601

[44] James E. Kelley Jr and Morgan R. Walker. 1959. Critical-path planning and scheduling. In Proceedings of the Eastern Joint IRE-AIEE-ACM Computer Conference (EJCC). 160-173. 
[45] Diederik P Kingma and Jimmy Ba. 2015. Adam: A method for stochastic optimization. Proceedings of the $7^{\text {th }}$ International Conference on Learning Representations (ICLR) (2015).

[46] Thomas N. Kipf and Max Welling. 2016. Semi-Supervised Classification with Graph Convolutional Networks. arXiv preprint arXiv:1609.02907 (2016). http://arxiv.org/abs/1609.02907

[47] Prasad A. Kulkarni. 2011. JIT compilation policy for modern machines. In ACM SIGPLAN Notices, Vol. 46. 773-788.

[48] Tom Leighton, Bruce Maggs, and Satish Rao. 1988. Universal packet routing algorithms. In Proceedings of the $29^{\text {th }}$ annual Symposium on Foundations of Computer Science (FOCS). 256-269.

[49] Zhuwen Li, Qifeng Chen, and Vladlen Koltun. 2018. Combinatorial optimization with graph convolutional networks and guided tree search. In Proceedings of the $32^{\text {nd }}$ Conference on Neural Information Processing Systems (NeurIPS). 539-548.

[50] Eric Liang and Richard Liaw. 2018. Scaling Multi-Agent Reinforcement Learning. https://bair.berkeley.edu/blog/2018/12/12/rllib/. (2018).

[51] Timothy P Lillicrap, Jonathan J Hunt, Alexander Pritzel, Nicolas Heess, Tom Erez, Yuval Tassa, David Silver, and Daan Wierstra. 2015. Continuous control with deep reinforcement learning. arXiv preprint arXiv:1509.02971 (2015).

[52] Chengzhi Lu, Kejiang Ye, Guoyao Xu, Cheng-Zhong Xu, and Tongxin Bai. 2017. Imbalance in the cloud: An analysis on alibaba cluster trace. In Proceedings of the 2017 IEEE International Conference on Big Data (BigData). IEEE, 2884-2892.

[53] Hongzi Mao, Mohammad Alizadeh, Ishai Menache, and Srikanth Kandula. 2016 Resource Management with Deep Reinforcement Learning. In Proceedings of the $15^{\text {th }}$ ACM Workshop on Hot Topics in Networks (HotNets).

[54] Hongzi Mao, Shannon Chen, Drew Dimmery, Shaun Singh, Drew Blaisdell, Yuandong Tian, Mohammad Alizadeh, and Eytan Bakshy. 2019. Real-world Video Adaptation with Reinforcement Learning. In Proceedings of the 2019 Reinforcement Learning for Real Life Workshop.

[55] Hongzi Mao, Shaileshh Bojja Venkatakrishnan, Malte Schwarzkopf, and Mohammad Alizadeh. 2019. Variance Reduction for Reinforcement Learning in Input-Driven Environments. Proceedings of the $7^{\text {th }}$ International Conference on Learning Representations (ICLR) (2019)

[56] Ryan Marcus, Parimarjan Negi, Hongzi Mao, Chi Zhang, Mohammad Alizadeh, Tim Kraska, Olga Papaemmanouil, and Nesime Tatbul. 2019. Neo: A Learned Query Optimizer. arXiv preprint arXiv:1904.03711 (2019)

[57] Monaldo Mastrolilli and Ola Svensson. 2008. (Acyclic) job shops are hard to approximate. In Proceedings of the $49^{\text {th }}$ IEEE Symposium on Foundations of Computer Science (FOCS). 583-592.

[58] Ishai Menache, Shie Mannor, and Nahum Shimkin. 2005. Basis function adaptation in temporal difference reinforcement learning. Annals of Operations Research 134, 1 (2005), 215-238.

[59] Azalia Mirhoseini, Anna Goldie, Hieu Pham, Benoit Steiner, Quoc V Le, and Jeff Dean. 2018. A Hierarchical Model for Device Placement. In Proceedings of the $6^{\text {th }}$ International Conference on Learning Representations (ICLR).

[60] Azalia Mirhoseini, Hieu Pham, Quoc V Le, Benoit Steiner, Rasmus Larsen, Yuefeng Zhou, Naveen Kumar, Mohammad Norouzi, Samy Bengio, and Jeff Dean. 2017. Device Placement Optimization with Reinforcement Learning. In Proceedings of the $33^{\text {rd }}$ International Conference on Machine Learning (ICML).

[61] Volodymyr Mnih, Koray Kavukcuoglu, David Silver, Andrei A. Rusu, Joel Veness, Marc G. Bellemare, Alex Graves, Martin Riedmiller, Andreas K. Fidjeland, Georg Ostrovski, Stig Petersen, Charles Beattie, Amir Sadik, Ioannis Antonoglou, Helen King, Dharshan Kumaran, Daan Wierstra, Shane Legg, Demis Hassabis, Ioannis Antonoglou, Daan Wierstra, and Martin A. Riedmiller. 2015. Human-level control through deep reinforcement learning. Nature 518 (2015), 529-533.

[62] Thanh Thi Nguyen, Ngoc Duy Nguyen, and Saeid Nahavandi. 2018. Deep Reinforcement Learning for Multi-Agent Systems: A Review of Challenges, Solutions and Applications. arXiv preprint arXiv:1812.11794 (2018).

[63] Kay Ousterhout, Ryan Rasti, Sylvia Ratnasamy, Scott Shenker, and Byung-Gon Chun. 2015. Making Sense of Performance in Data Analytics Frameworks. In Proceedings of the $12^{\text {th }}$ USENIX Symposium on Networked Systems Design and Implementation (NSDI). 293-307. https: //www.usenix.org/conference/nsdi15/technical-sessions/presentation/ousterhout

[64] Lerrel Pinto, James Davidson, Rahul Sukthankar, and Abhinav Gupta. 2017. Robust Adversarial Reinforcement Learning. In Proceedings of the $34^{\text {th }}$ International Conference on Machine Learning (ICML). 2817-2826.

[65] Chandrasekharan Rajendran. 1994. A no-wait flowshop scheduling heuristic to minimize makespan. Journal of the Operational Research Society 45, 4 (1994), 472-478.

[66] Jeff Rasley, Konstantinos Karanasos, Srikanth Kandula, Rodrigo Fonseca, Milan Vojnovic, and Sriram Rao. 2016. Efficient Queue Management for Cluster Scheduling. In Proceedings of the $11^{\text {th }}$ European Conference on Computer Systems (EuroSys). Article 36, 15 pages. https://doi.org/10.1145/2901318.2901354

[67] John Schulman, Sergey Levine, Pieter Abbeel, Michael Jordan, and Philipp Moritz. 2015. Trust region policy optimization. In Proceedings of the 32nd International Conference on Machine Learning (ICML). 1889-1897.
[68] Malte Schwarzkopf, Andy Konwinski, Michael Abd-El-Malek, and John Wilkes. 2013. Omega: flexible, scalable schedulers for large compute clusters. In Proceedings of the $8^{\text {th }}$ ACM European Conference on Computer Systems (EuroSys). 351-364.

[69] David B Shmoys, Clifford Stein, and Joel Wein. 1994. Improved approximation algorithms for shop scheduling problems. SIAM J. Comput. 23, 3 (1994), 617-632.

[70] David Silver, Aja Huang, Christopher J. Maddison, Arthur Guez, Laurent Sifre, George van den Driessche, Julian Schrittwieser, Ioannis Antonoglou, Veda Panneershelvam, Marc Lanctot, Sander Dieleman, Dominik Grewe, John Nham, Nal Kalchbrenner, Ilya Sutskever, Timothy Lillicrap, Madeleine Leach, Koray Kavukcuoglu, Thore Graepel, and Demis Hassabis. 2016. Mastering the game of Go with deep neural networks and tree search. Nature 529 (2016), 484-503.

[71] David Silver, Julian Schrittwieser, Karen Simonyan, Ioannis Antonoglou, Aja Huang, Arthur Guez, Thomas Hubert, Lucas Baker, Matthew Lai, Adrian Bolton, Yutian Chen, Timothy Lillicrap, Fan Hui, Laurent Sifre, George van den Driessche, Thore Graepel, and Demis Hassabis. 2017. Mastering the game of Go without human knowledge. Nature 550, 7676 (2017), 354.

[72] Richard. S. Sutton and Andrew. G. Barto. 2017. Reinforcement Learning: An Introduction, Second Edition. MIT Press.

[73] TPC-H 2018. The TPC-H Benchmarks. www.tpc.org/tpch/. (2018).

[74] Alexey Tumanov, Timothy Zhu, Jun Woo Park, Michael A. Kozuch, Mor Harchol-Balter, and Gregory R. Ganger. 2016. TetriSched: Global Rescheduling with Adaptive Plan-ahead in Dynamic Heterogeneous Clusters. In Proceedings of the $11^{\text {th }}$ European Conference on Computer Systems (EuroSys). Article 35, 16 pages. https://doi.org/10.1145/2901318.2901355

[75] Vinod Kumar Vavilapalli, Arun C. Murthy, Chris Douglas, Sharad Agarwal, Mahadev Konar, Robert Evans, Thomas Graves, Jason Lowe, Hitesh Shah, Siddharth Seth, Bikas Saha, Carlo Curino, Owen O’Malley, Sanjay Radia, Benjamin Reed, and Eric Baldeschwieler. 2013. Apache Hadoop YARN: Yet Another Resource Negotiator. In Proceedings of the $4^{\text {th }}$ annual Symposium on Cloud Computing (SoCC). Article 5, 16 pages. https://doi.org/10.1145/2523616.2523633

[76] Abhishek Verma, Madhukar Korupolu, and John Wilkes. 2014. Evaluating job packing in warehouse-scale computing. In Proceedings of the 2014 IEEE International Conference on Cluster Computing (CLUSTER). 48-56.

[77] Abhishek Verma, Luis Pedrosa, Madhukar R. Korupolu, David Oppenheimer, Eric Tune, and John Wilkes. 2015. Large-scale cluster management at Google with Borg. In Proceedings of the $10^{\text {th }}$ European Conference on Computer Systems (EuroSys). Bordeaux, France.

[78] Lex Weaver and Nigel Tao. 2001. The optimal reward baseline for gradient-based reinforcement learning. In Proceedings of the $17^{\text {th }}$ Conference on Uncertainty in Artificial Intelligence (UAI). 538-545.

[79] Ronald J Williams. 1992. Simple statistical gradient-following algorithms for connectionist reinforcement learning. Machine learning 8, 3-4 (1992), 229-256.

[80] Matei Zaharia, Mosharaf Chowdhury, Tathagata Das, Ankur Dave, Justin Ma, Murphy McCauley, Michael J. Franklin, Scott Shenker, and Ion Stoica. 2012. Resilient Distributed Datasets: A Fault-tolerant Abstraction for In-memory Cluster Computing. In Proceedings of the $9^{\text {th }}$ USENIX Conference on Networked Systems Design and Implementation (NSDI). 15-28. http://dl.acm.org/citation.cfm?id=2228298.2228301 


\section{Appendices}

Appendices are supporting material that has not been peer reviewed.

\section{A An example of dependency-aware scheduling}
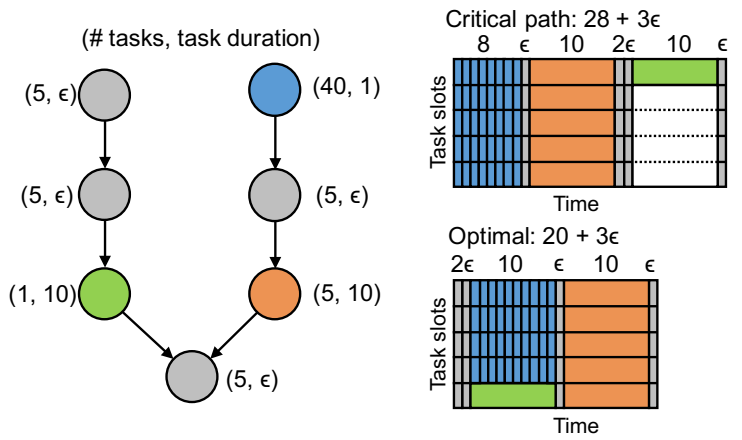

Figure 16: An optimal DAG-aware schedule plans ahead and parallelizes execution of the blue and green stages, so that orange and green stages complete at the same time and the bottom join stage can execute immediately. A straightforward critical path heuristic would instead focus on the right branch, and takes $29 \%$ longer to execute the job.

Directed acyclic graphs (DAGs) of dependent operators or "stages" are common in parallel data processing applications. Figure 16 shows a common example: a DAG with two branches that converge in a join stage. A simple critical path heuristic would choose to work on the right branch, which contains more aggregate work: 90 task-seconds vs. 10 task-seconds in the left branch. With this choice, once the orange stage finishes, however, the final join stage cannot run, since its other parent stage (in green) is still incomplete. Completing the green stage next, followed by the join stage - as a critical-path schedule would results in an overall makespan of $28+3 \epsilon$. The optimal schedule, by contrast, completes this DAG in $20+3 \epsilon$ time, $29 \%$ faster. Intuitively, an ideal schedule allocates resources such that both branches reach the final join stage at the same time, and execute it without blocking.

\section{B Background on Reinforcement Learning}

We briefly review reinforcement learning (RL) techniques that we use in this paper; for a detailed survey and rigorous derivations, see e.g., Sutton and Barto's book [72].

Reinforcement learning. Consider the general setting in Figure 17, where an RL agent interacts with an environment. At each step $k$, the agent observes some state $s_{k}$, and takes an action $a_{k}$. Following the action, the state of the environment transitions to $s_{k+1}$ and the agent receives a reward $r_{k}$ as feedback. The state transitions and rewards are stochastic and assumed to be a Markov process: the state transition to $s_{k+1}$ and the reward $r_{k}$ depend only on the state $s_{k}$ and the action $a_{k}$ at step $k$ (i.e., they are conditionally independent of the past).

In the general RL setting, the agent only controls its actions: it has no a priori knowledge of the state transition probabilities or the reward function. However, by interacting with the environment, the agent can learn these quantities during training.

For training, RL proceeds in episodes. Each episode consists of a sequence of (state, action, reward) observations - i.e., $\left(s_{k}, a_{k}, r_{k}\right)$ at each step $k \in[0,1, \ldots, T]$, where $T$ is the episode length . For ease of understanding, we first describe an RL formulation that maximizes

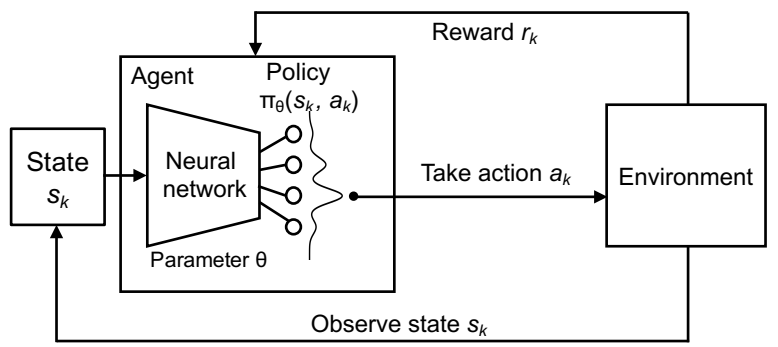

Figure 17: A reinforcement learning setting with neural networks [53, 72]. The policy is parameterized using a neural network and is trained iteratively via interactions with the environment that observe its state and take actions.

the total reward: $\mathbb{E}\left[\sum_{k=0}^{T} r_{k}\right]$. However, in our scheduling problem, the average reward formulation (\$5.3) is more suitable. We later describe how to modify the reward signal to convert the objective to the average reward setting.

Policy. The agent picks actions based on a policy $\pi\left(s_{k}, a_{k}\right)$, defined as a probability of taking action $a_{k}$ at state $s_{k}$. In most practical problems, the number of possible \{state, action $\}$ pairs is too large to store the policy in a lookup table. It is therefore common to use function approximators $[15,58]$, with a manageable number of adjustable parameters, $\theta$, to represent the policy as $\pi_{\theta}\left(s_{k}, a_{k}\right)$. Many forms of function approximators can be used to represent the policy. Popular choices include linear combinations of features of the state/action space (i.e., $\pi_{\theta}\left(s_{k}, a_{k}\right)=\theta^{T} \phi\left(s_{k}, a_{k}\right)$ ), and, recently, neural networks [39] for solve large-scale RL tasks [61, 71]. An advantage of neural networks is that they do not need hand-crafted features, and that they are end-to-end differentiable for training.

Policy gradient methods. We focus on a class of RL algorithms that perform training by using gradient-descent on the policy parameters. Recall that the objective is to maximize the expected total reward; the gradient of this objective is given by:

$$
\nabla_{\theta} \mathbb{E}_{\pi_{\theta}}\left[\sum_{k=0}^{T} r_{k}\right]=\mathbb{E}_{\pi_{\theta}}\left[\sum_{k=0}^{T} \nabla_{\theta} \log \pi_{\theta}\left(s_{k}, a_{k}\right) Q^{\pi_{\theta}}\left(s_{k}, a_{k}\right)\right],
$$

where $Q^{\pi_{\theta}}\left(s_{k}, a_{k}\right)$ is the expected total discounted reward from (deterministically) choosing action $a_{k}$ in state $s_{k}$, and subsequently following policy $\pi_{\theta}[72, \S 13.2]$. The key idea in policy gradient methods is to estimate the gradient using the trajectories of execution with the current policy. Following the Monte Carlo Method [40], the agent samples multiple trajectories and uses the empirical total discounted reward, $v_{k}$, as an unbiased estimate of $Q^{\pi_{\theta}}\left(s_{k}, a_{k}\right)$. It then updates the policy parameters via gradient descent:

$$
\theta \leftarrow \theta+\alpha \sum_{k=0}^{T} \nabla_{\theta} \log \pi_{\theta}\left(s_{k}, a_{k}\right) v_{k},
$$

where $\alpha$ is the learning rate. This equation results in the REINFORCE algorithm [79]. The intuition of REINFORCE is that the direction $\nabla_{\theta} \log \pi_{\theta}\left(s_{k}, a_{k}\right)$ indicates how to change the policy parameters in order to increase $\pi_{\theta}\left(s_{k}, a_{k}\right)$ (the probability of action $a_{k}$ at state $s_{k}$ ). Equation 5 takes a step in this direction; the size of the step depends on the magnitude of the return $v_{k}$. The net effect is to reinforce actions 
that empirically lead to better returns. Appendix $\mathrm{C}$ describes how we implement this training method in practice.

Policy gradient methods are better suited to our scheduling context than the alternative value-based methods for two reasons. First, policybased methods are easier to design if it is unclear whether the neural network architecture used has adequate expressive power. The reason is that value-based methods aim to find a fixed-point of the Bellman equations [13]. If the underlying neural network cannot express the optimal value function, then a value-based method can have difficulty converging because the algorithm is trying to converge to a fixed point that the neural network cannot express. With policy-based methods, this issue does not arise, because regardless of the policy network's expressive power, the policy gradient algorithm will optimize for the reward objective over the space of policies that the neural network can express. Second, policy gradient methods allow us to use inputdependent baselines [55] to reduce training variance (challenge \#2 in $\S 5.3$ ). It is currently unknown whether, and how, this technique can be applied to value-based methods.

Average reward formulation. For our scheduling problem, an average reward formulation, which maximizes $\lim _{T \rightarrow \infty} \mathbb{E}\left[1 / T \sum_{k=0}^{T} r_{k}\right]$, is a better objective than the total reward we discussed so far.

To convert the objective from the sum of rewards to the average reward, we replace the reward $r_{k}$ with a differential reward. Operationally, at every step $k$, the environment modifies the reward to the agent as $r_{k} \leftarrow r_{k}-\hat{r}$, where $\hat{r}$ is a moving average of the rewards across a large number of previous steps (across many training episodes). With this modification, we can reuse the same policy gradient method as in Equation (4) and (5) to find the optimal policy. Sutton and Barto [72, $\S 10.3, \S 13.6$ ] describe the mathematical details on how this approach optimizes the average reward objective.

\section{Training implementation details}

Algorithm 1 presents the pseudocode for Decima's training procedure as described in §5.3. In particular, line 3 samples the episode length $\tau$ from an exponential distribution, with a small initial mean $\tau_{\text {mean }}$. This step terminates the initial episodes early to avoid wasting training time (see challenge \#1 in §5.3). Then, we sample a job sequence (line 4 ) and use it to collect $N$ episodes of experience (line 5). Importantly, the baseline $b_{k}$ in line 8 is computed with the same job sequence to reduce the variance caused by the randomness in the job arrival process (see challenge \#2 in $\S 5.3$ ). Line 10 is the policy gradient REINFORCE algorithm described in Eq. (3). Line 13 increases the average episode length (i.e., the curriculum learning procedure for challenge \#1 in §5.3). Finally, we update Decima’s policy parameter $\theta$ on line 14.

Our neural network architecture is described in $\$ 6.1$, and we set the hyperparameters in Decima's training as follows. The number of incoming jobs is capped at 2000 , and the episode termination probability decays linearly from $5 \times 10^{-7}$ to $5 \times 10^{-8}$ throughout training. The learning rate $\alpha$ is $1 \times 10^{-3}$ and we use Adam optimizer [45] for gradient descent. For continuous job arrivals, the moving window for estimating $\hat{r}$ spans $10^{5}$ time steps (see the average reward formulation in Appendix B). Finally, we train Decima for at least 50,000 iterations for all experiments.

We implemented Decima's training framework using TensorFlow [1], and we use 16 workers to compute episodes with the same job sequence in parallel during training. Each training iteration, including

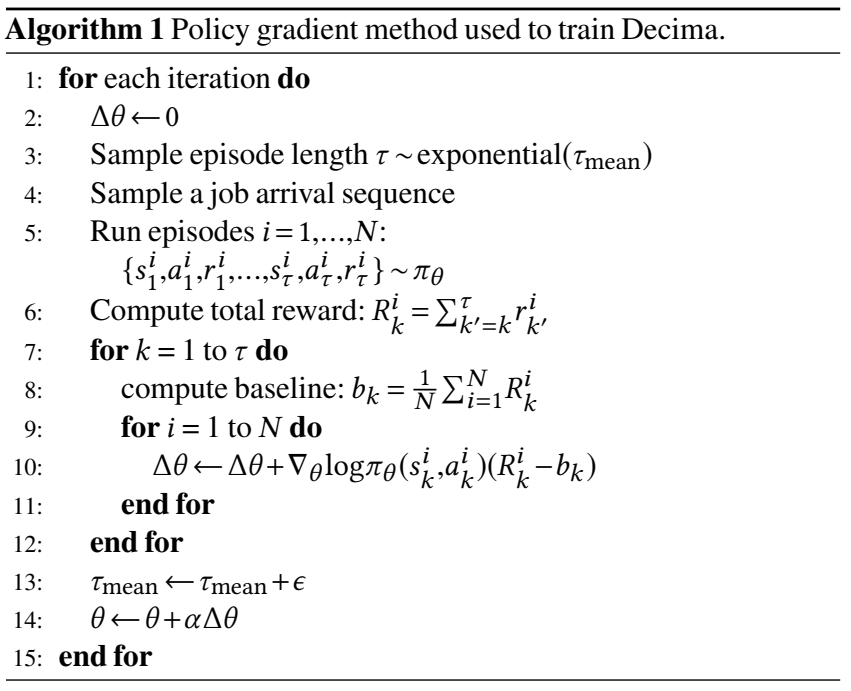

interaction with the simulator, model inference and model update from all training workers, takes roughly 1.5 seconds on a machine with Intel Xeon E5-2640 CPU and Nvidia Tesla P100 GPU.

All experiments in $\$ 7$ are performed on test job sequences unseen during training (e.g., unseen TPC-H job combinations, unseen part of the Alibaba production trace, etc.).

\section{Simulator fidelity}

Our training infrastructure relies on a faithful simulator of Spark job execution in a cluster. To validate the simulator's fidelity, we measured how simulated and real Spark differ in terms of job completion time for ten runs of TPC-H job sets (\$7.2), both when jobs run alone and when they share a cluster with other jobs. Figure 18 shows the results: the simulator closely matches the actual run time of each job, even when we run multiple jobs together in the cluster. In particular, the mean error of our simulation is within $5 \%$ of real runtime when jobs run in isolation, and within $9 \%$ when sharing a cluster $\left(95^{\text {th }}\right.$ percentile: $\leq 10 \%$ in isolation, $\leq 20 \%$ when sharing).

We found that capturing all first-order effects of the Spark environment is crucial to achieving this accuracy (\$6.2). For example, without modeling the delay to move an executor between jobs, the simulated runtime consistently underapproximates reality. Training in such an environment would result in a policy that moves executors more eagerly than is actually sensible (\$7.4). Likewise, omitting the effects of initial and subsequent "waves" of tasks, or the slowdown overheads imposed with high degrees of paralllism, significantly increases the variance in simulated runtime and makes it more difficult for Decima to learn a good policy.

\section{E Expressiveness of Decima's state representation}

Decima's can only learn strong scheduling policies if its state representation, embedding scheme, and neural network architecture can express them (\$7).

In Equation (1), combining two non-linear transforms $f(\cdot)$ and $g(\cdot)$ enables Decima to express a wide variety of aggregation functions. For example, if $f \sim \log (\cdot / n), g \sim \exp (n \times \cdot)$, and $n \rightarrow \infty$, the aggregation computes the maximum of the child node embeddings. By contrast, a standard aggregation operation of the form $\mathbf{e}_{\mathbf{v}}=\sum_{u \in \xi(v)} f\left(\mathbf{e}_{\mathbf{u}}\right)$ 


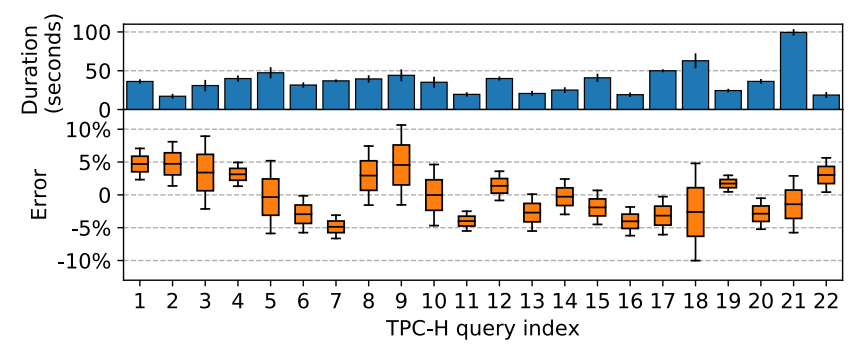

(a) Single job running in isolation.

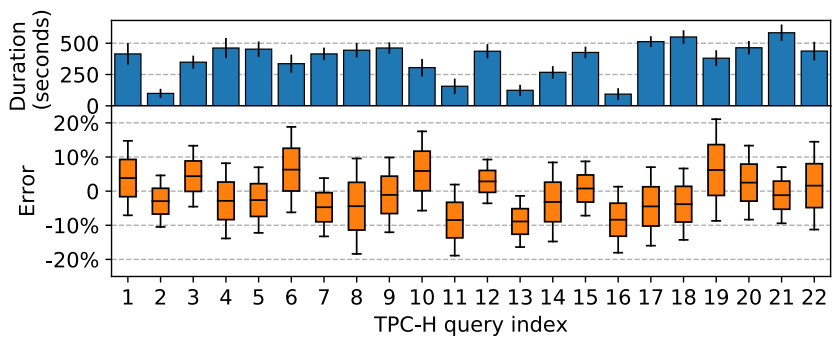

(b) Mixture of jobs on a shared cluster.

Figure 18: Testing the fidelity of our Spark simulator with Decima as a scheduling agent. Blue bars in the upper part show the absolute real Spark job duration (error bars: standard deviation across ten experiments); the orange bars in the lower figures show the distribution of simulation error for a $95 \%$ confidence interval. The mean discrepancy between simulated and actual job duration is at most $\pm 5 \%$ for isolated, single jobs, and the mean error for a mix of all 22 queries running on the cluster is at most $\pm 9 \%$.

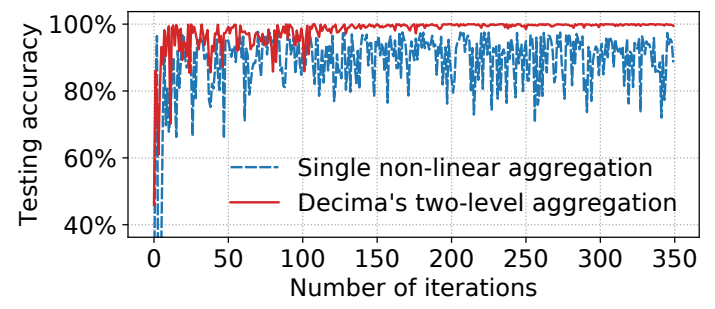

Figure 19: Trained using supervised learning, Decima's two-level non-linear transformation is able to express the max operation necessary for computing the critical path (\$5.1), and consequently achieves near-perfect accuracy on unseen DAGs compared to the standard graph embedding scheme.

without a second non-linear transformation $g(\cdot)$ is insufficient to express the max operation. Consequently, such an architecture cannot learn the aggregation $(\max )$ required to find the critical path of a graph.

During development, we relied on a simple sanity check to test the expressiveness of a graph embedding scheme. We used supervised learning to train the graph neural network to output the critical path value of each node in a large number of random graphs, and then checked how accurately the graph neural network identified the node with the maximum critical path value. Figure 19 shows the testing accuracy that Decima's node embedding with two aggregation levels achieves on unseen graphs, and compares it to the accuracy achieved by a simple, single-level embedding with only one nonlinear transformation. Decima's node embedding manages to learn the max operation and therefore accurately identifies the critical path after about 150 iterations, while the standard embedding is incapable of expressing the critical path and consequently never reaches a stable high accuracy.

\section{F Multi-resource scheduling heuristic comparison details}

When evaluating Decima's performance in a multi-resource setting (§7.3), we compared with several heuristics.

First, we considered the optimally tuned weighted fair heuristic from $\$ 7.2$. This heuristic grants each job an executor share based on the total work in the job. Then the heuristic chooses a stage the same way as in the single resource setting. Among the available executor types, the heuristic first exhausts the best-fitting category before choosing any others. The scheduler ensures that the aggregate allocated resources (across different executor types) do not exceed the job's weighted fair share.

Second, we compared to the resource-packing algorithm from Tetris [34]. To maximize resource utilization, we select the DAG node that yields the largest dot product of the requested resource vector and the available resource vector for each executor type. Then, we greedily grant as much parallelism as the tasks in this node need.

The prior two heuristics lack each other's key scheduling ingredients (fairness and packing), and neither understands the DAG structure. Finally, we compared to Graphene [36], whose hybrid heuristic combines these factors. However, our multi-resource scheduling environment with discrete executor classes differs from the original Graphene setting, which assumes continuous, infinitely divisible resources. We adapted the Graphene algorithm for discrete executors, but kept its essence: specifically, we estimate and group the "troublesome" nodes the same way [36, §4.1]. To ensure that troublesome nodes are scheduled at the same time, we dynamically suppress the priority on all troublesome nodes of a DAG until all of these nodes are available in the frontier. We also include parallelism control by sharing the executors according to the optimally tuned weighted fair partition heuristic; and we pack resources by prioritizing the executor type that best fits the resource request. Finally, we perform a grid search on all the hyperparameters (e.g., the threshold for picking troublesome nodes) to tune the heuristic for the best scheduling performance in each of the experiments $(\$ 7.3)$.

\section{G Further analysis of multi-resource scheduling}

In $\$ 7.3$, we found that Decima achieves $32 \%$ - 43\% lower average JCT than state-of-the-art heuristics when handling continuous job arrivals in a multi-resource environment. Decima achieves this by carefully fragmenting cluster memory: Figure $12 \mathrm{~b}$ illustrated that Decima selectively borrows large executors if they can help finishing short jobs quickly and increase cluster throughput.

This effect is also evident when examining the timeseries of job duration and executor usage over a single experiment. Figure 20 shows that Decima maintains a smaller number of concurrent active jobs during periods of high load both for a synthetic TPC-H workload and for the Alibaba trace. During busy periods (e.g., around snapshot 50 in Figure 20a1), Decima clears the backlog of jobs in the queue more quickly than the best competing heuristic, Graphene*. During these periods, Decima assigns more executors to each job than Graphene* (Figures 20a2 and 20b2), e.g., by sometimes borrowing large executors for jobs that need only smaller ones. As a consequence, Decima 

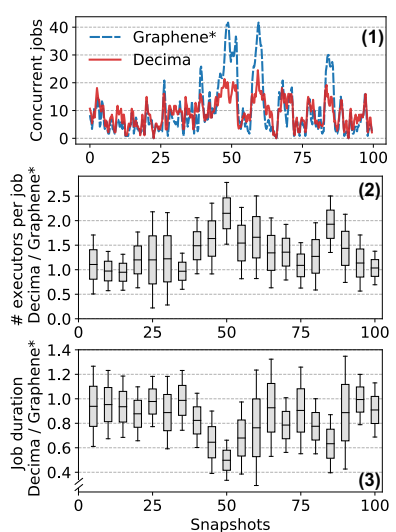

(a) TPC-H workload.
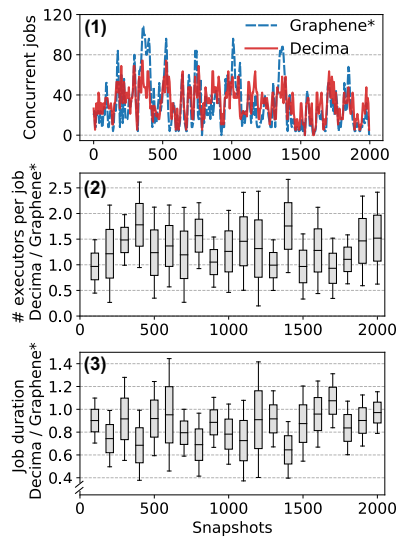

(b) Industrial trace replay.

Figure 20: Timeseries of different statistics in the extended Spark multiresource environment. We compare Decima and Graphene*, the best competing heuristic. During busy periods, Decima finishes jobs faster and maintains a lower number of concurrent jobs by using more executors per job.

achieves lower JCT and higher cluster throughput when the cluster load is high (Figures 20a3 and 20b3).

Figure $21 \mathrm{a}$ and $21 \mathrm{~b}$ compare the executor assignment between Decima and Graphene* in detail (Figure 12b is the first column of this profile). On the $x$-axis, we bin jobs according to the total amount of work they contain (in task-seconds). The $y$-axis of each graph is the number of executors Decima uses normalized to the number of executors used by Graphene* — i.e., a value above one indicates that Decima used more executors. Overall, Decima tends to assign more executors per job compared to Graphene*. This helps Decima complete jobs faster in order to then move on to others, instead of making progress on many jobs concurrently, similar to the behavior we discussed in §7.2. Moreover, Decima uses more large executors on small jobs. This aggressive allocation of large executors - which wastes some memory - leads to faster job completion during the busy periods (Figure 20a3 and 20b3), at the expense of leaving some memory unused. This trade-off between resource fragmentation and prioritizing small jobs can be tedious to balance, but Decima automatically learns a strong policy by interacting with the environment.

Decima may enjoy an advantage here partly because Graphene* is restricted to discrete executor classes. In a cluster setting with arbitrary, continuous memory assignment to tasks, large executor "slots" could be subdivided into multiple smaller executors, assuming sufficient CPU capacity exists. This choice is difficult to express with a finite action space like Decima's, and it is an interesting direction for future work to investigate whether RL with continuous action could be applied to cluster scheduling.

\section{H Optimality of Decima}

In $\S 7$, we show Decima is able to rival or outperform existing scheduling schemes in a wide range of complex cluster environments, including a real Spark testbed, real-world cluster trace simulations and a multi-resource packing environment. However, the optimality of Decima in those environments remains unknown due to the intractability of computing exact optimal scheduling solutions [36, 57], or tight
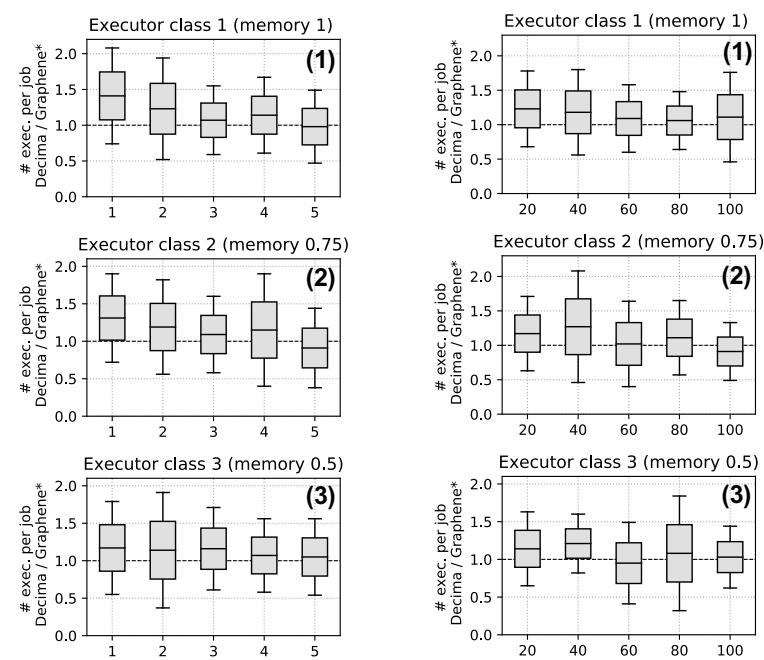

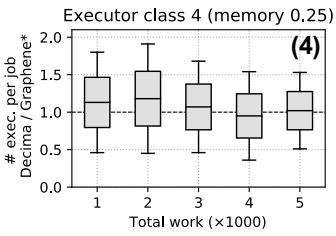

(a) TPC-H workload.

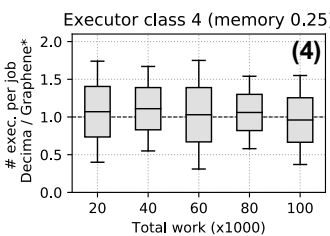

(b) Industrial trace replay.
Figure 21: Profile of executor assignments on jobs with different sizes, Decima normalized to Graphene*'s assignment ( $>1$ : more executors in Decima, <1: more in Graphene*). Decima tends to assign more executors.

lower bounds. ${ }^{9}$ To nevertheless get an idea of how close Decima comes to an optimal scheduler, we test Decima in simplified settings where a brute-force search over different schedules is possible.

We consider the Spark scheduling framework simulated in $\$ 6.2$ with an average JCT objective for a batch of jobs. To simplify the environment, we turn off the "wave" effect, executor startup delays and the artifact of task slowdowns at high degrees of parallelism. As a result, the duration of a stage has a strict inverse relation to the number of executors the stage runs on (i.e., it scales linearly with parallel resources), and the scheduler is free to move executors across jobs without any overhead. The dominating challenges in this environment are to pack jobs tightly and to favor short jobs as much as possible.

To find a good schedule for a batch of $n$ jobs, we exhaustively search all $n$ ! possible job orderings, and select the ordering with the lowest average JCT. To make the exhaustive search feasible, we consider a batch of ten jobs. For each job ordering, we select the unfinished job appearing earliest in the order at each scheduling event (\$5.2), and use the DAG's critical path to choose the order in which to finish stages within each job. By considering all possible job orderings, the algorithm is guaranteed to consider, amongst other schedules, a strict shortest-job-first (SJF) schedule that yields a small average JCT. We believe this policy to be close to the optimal policy, as we have empirically observed that job orderings dominate the average JCT in TPC-H workloads (\$7.4). However, the exhaustive search also explores variations of the SJF schedule, e.g., orders that prioritize

\footnotetext{
${ }^{9}$ In our setting (i.e., Spark's executor-based scheduling), we found lower bounds based on total work or the critical path to be too loose to provide meaningful information.
} 


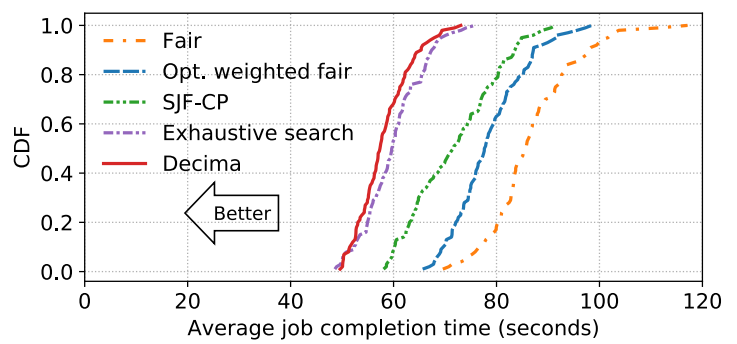

Figure 22: Comparing Decima with near optimal heuristics in a simplified scheduling environment.

\begin{tabular}{|l|c|}
\hline Decima training scenario & average JCT (seconds) \\
\hline Decima trained with test setting & $3,290 \pm 680$ \\
Decima trained with $15 \times$ fewer jobs & $3,540 \pm 450$ \\
\hline Decima trained with test setting & $610 \pm 90$ \\
Decima trained with $10 \times$ fewer executors & $630 \pm 70$ \\
\hline
\end{tabular}

Table 3: Decima generalizes well to deployment scenarios in which the workload or cluster differ from the training setting. The test setting has 150 jobs and 10k executors.

jobs which can exploit parallelism to complete more quickly than less-parallelizable jobs that contain smaller total work.

Next, we train an unmodified Decima agent in this environment, similar to the setup in $\$ 7.2$. We compare this agent's performance with our exhaustive search baseline, a shortest-job-first critical-path heuristic, and the tuned weighted fair scheduler (described in \$7.2).

Figure 22 shows the results. We make three key observations. First, unlike in the real Spark cluster (Figure 9), the SJF-CP scheme outperforms the tuned weighted fair scheduler. This meets our expectation because SJF-CP strictly favors small jobs to minimize the average JCT, which in the absence of the complexities of a real-world cluster is a good policy. Second, the exhaustive search heuristic performs better than SJF-CP. This is because SJF-CP strictly focuses on completing the job with the smallest total work first, ignoring the DAG structure and the potential parallelism it implies. The exhaustive search, by contrast, finds job orderings that prioritize jobs which can execute most quickly given the available executors on the cluster, their DAG structure, and their total work. While the search algorithm is not aware of these constraints, by trying out different job orderings, it finds the schedule that both orders jobs correctly and exploits cluster resources to complete the jobs as quickly as possible. Third, Decima matches the average JCT of the exhaustive search or even outperforms it slightly (by $9 \%$ on average). We found that Decima is better at dynamically prioritizing jobs based on their current structure at runtime (e.g., how much work remains on each dependency path), while the exhaustive search heuristic strictly follows the order determined in an offline static search and only controls when jobs start. This experiment shows that Decima is able to automatically learn a scheduling algorithm that performs as well as an offline-optimal job order.

\section{Generalizing Decima to different environments}

Real-world cluster workloads vary over time, and the available cluster machines can also change. Ideally, Decima would generalize from a

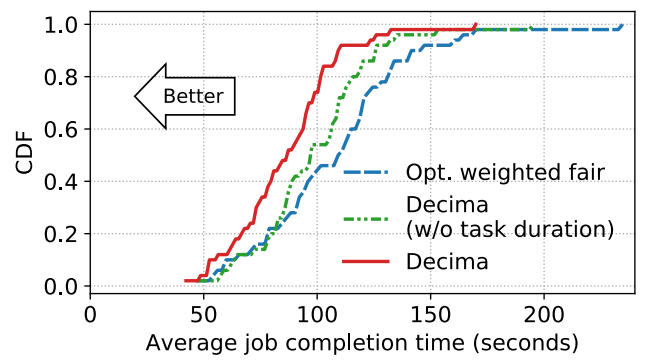

Figure 23: Decima performs worse on unseen jobs without task duration estimates, but still outperforms the best heuristic.

model trained for a specific load and cluster size to similar workloads with different parameters. To test this, we train a Decima agent on a scaled-down version of the industrial workload, using $15 \times$ fewer concurrent jobs and $10 \times$ fewer executors than in the test setting.

Table 3 shows how the performance of this agent compares with that of one trained on the real workload and cluster size. Decima is robust to changing parameters: the agent trained with $15 \times$ fewer jobs generalizes to the test workload with a $7 \%$ reduced average JCT, and an agent trained on a $10 \times$ smaller cluster generalizes with a $3 \%$ reduction in average JCT. Generalization to a larger cluster is robust as the policy correctly limits jobs' parallelism even if vastly more resources are available. By contrast, generalizing to a workload with many more jobs is harder, as the smaller-scale training lacks experiences with complex job combinations.

\section{J Decima with incomplete information}

In a real cluster, Decima will occasionally encounter unseen jobs without reliable task duration estimates. Unlike heuristics that fundamentally rely on profiling information (e.g., weighted fair scheduling based on total work), Decima can still work with the remaining information and extract a reasonable scheduling policy.

Running the same setting as in $\S 7.2$, Figure 23 shows that training without task durations yields a policy that still outperforms the best heuristic, as Decima can still exploit the graph structure and other information such as the correlation between number of tasks and the efficient parallelism level. 